\title{
Recently evolved Francisellalike endosymbiont outcompetes an ancient and evolutionarily associated Coxiellalike endosymbiont in the lone star tick (Amblyomma americanum) linked to the Alpha-Gal Syndrome
}

\section{Deepak Kumar}

University of Southern Mississippi Center For Tobacco Prevention and Health Promotion: University of Southern Mississippi

\section{Surendra Raj Sharma}

University of Southern Mississippi Center For Tobacco Prevention and Health Promotion: University of Southern Mississippi

\section{Abdulsalam Adegoke}

University of Southern Mississippi

\section{Ashley Kennedy}

Delaware Division of Fish and Wildlife

Holly C. Tuten

University of Illinois at Urbana-Champaign

Andrew Y. Li

USDA ARS: USDA Agricultural Research Service

Shahid Karim (D Shahid.Karim@usm.edu )

University of Southern Mississippi https://orcid.org/0000-0001-5596-3304

\section{Research Article}

Keywords: Alpha-Gal Syndrome, Amblyomma americanum, endosymbionts, lone star tick, microbiome, ticks

Posted Date: September 3rd, 2021

DOI: https://doi.org/10.21203/rs.3.rs-856007/v1

License: (c) (i) This work is licensed under a Creative Commons Attribution 4.0 International License. Read Full License 
Version of Record: A version of this preprint was published at Frontiers in Cellular and Infection Microbiology on April 12th, 2022. See the published version at https://doi.org/10.3389/fcimb.2022.787209. 


\section{Abstract}

\section{Background}

Ticks are hematophagous arthropods that transmit various bacterial, viral, and protozoan pathogens of public health significance. The lone star tick (Amblyomma americanum) is an aggressive human-biting tick that transmits bacterial and viral pathogens, and its bites are suspected of eliciting the Alpha-Gal Syndrome, a newly emerged delayed hypersensitivity following consumption of red meat in the United States. While ongoing studies have attempted to investigate the contribution of different tick-inherent factors to the induction of Alpha-Gal syndrome, an otherwise understudied aspect is the contribution of the tick microbiome and specifically obligate endosymbionts to the establishment of the Alpha-Gal syndrome in humans.

\section{Methods}

Here we utilized a high throughput metagenomic sequencing approach to cataloging the entire microbial communities residing within different developmental stages and tissues of unfed and blood-fed ticks from laboratory-maintained ticks and three new geographical locations in the USA. The quantitative insights into microbial ecology (QIIME2) pipeline were used to perform data analysis and taxonomic classification. Moreover, using a SparCC network construction model, we investigated potential interactions between members of the microbial communities from lab-maintained and field-collected ticks.

\section{Results}

Overall, Francisellaceae was the most dominant bacteria identified in the microbiome of both lab-raised and field-collected Am. americanum across all tissues and developmental stages. Likewise, microbial diversity was seen to be significantly higher in field-collected ticks compared to lab-maintained ticks as seen with a higher number of both OTUs and measures of species richness. Several potential positive and negative correlations were identified from our network analysis. We observed a strong positive correlation between Francisellaceae, Rickettsiaceae, and Midichloriaceae in both developmental stages and tissues from lab-maintained ticks, while ovarian tissues had a strong positive correlation of bacteria in the family Xanthobacteraceae and Rhizobiaceae. A negative interaction was observed between Coxiellaceae and Francisellaceae in Illinois, and all the bacteria detected from ticks from Delaware were negatively correlated.

\section{Conclusion}

This study is the first to catalog the microbiome of Am. americanum throughout its developmental stages and different tissue niches and report the potential replacement of Coxiellaceae by Francisellaceae across developmental stages and tissues tested except in ovarian tissues. These unique and significant findings advance our knowledge and open a new avenue of research to further understand the role of tick microbiome in tick-borne diseases and develop a holistic strategy to control Alpha-Gal syndrome. 


\section{Background}

The lone star tick (Amblyomma americanum) (Linnaeus, 1758) is an aggressive and generalist hematophagous species known as a vector of a variety of viral (Heartland virus) and bacterial pathogens (Ehrlichia chaffeensis, E. ewingii, Francisella tularensis, and Borrelia lonestari), and Alpha-Gal Syndrome [1]. Microorganisms that occupy an arthropod tick vector are collectively called the tick microbiome; however, the collection of commensals, symbiotic, and pathogenic microbes associated with ticks is more precisely termed the "pathobiome". Past investigations of the pathobiome of the lone star tick primarily focused on field-collected tick samples (nymphs or adults) using the 16S ribosomal RNA (16S rRNA) sequencing approach [2-5]. Microbes co-exiting association with known pathogens within the ticks might influence pathogen infection and transmission. For instance, rickettsial endosymbionts are thought to alter the transmission of other rickettsial pathogens, as reported by the inverse relationship between the infection prevalence of Rickettsia rickettsii (pathogen) and $R$. peacockii (symbiont) in Dermacentor andersoni [6]. Similarly, the presence of Coxiella-related symbionts in the salivary glands of Amblyomma americanum ticks impairs the transmission of Ehrlichia chaffeensis [7]. In addition to symbionts, ticks maintain a natural bacterial flora predominantly comprised of bacteria from the Proteobacteria, Firmicutes, and Bacteroides phyla [8-13], which have also been implicated in interference with tick pathogen infection. For example, Ixodes scapularis ticks hatched and raised in a sterile environment showed an altered microbiota, impaired gut integrity, and a reduced ability for Borrelia burgdorferi to colonize [14]. As demonstrated in other arthropod vectors [15], altering the tick microbiome may also result in a modulated immune response that could also interfere with pathogen infection and transmission. Since microbiomes are an integral part of tick biology, a detailed understanding of 1) microbiome composition, 2) stability, and 3) tripartite interaction with the tick, pathogen, and host will facilitate the discovery of new interventional strategies to limit tick-borne diseases (TBD).

Most microbiome studies have been performed in whole ticks except in a few studies where tick tissues have been used $[8,14,16-18]$. Microbiome profiling using whole ticks has limitations, such as difficulty profiling a specific microbial niche at the tissue level [19]. Profiling of the microbiome associated with vital tissues is necessary for deriving functional inferences of tick biology, tick-borne diseases, and interactions (e.g., tick-pathogen, pathogen-endosymbiont). Furthermore, exoskeleton-associated environmental contaminants could may not be differentiated from bona fide tick tissue- associated bacteria [13]. Previous studies have also shed light on the prevalence of certain endosymbionts within tick samples. A previously published study showed that around $1 / 3$ of the tick species examined either lacked Coxiella-like endosymbiont (CLE) or harbored lower frequencies than expected of an obligate endosymbiont [20-21]. Tick species deficient in CLE are scattered among major tick families and genera, suggesting its frequent and repeated loss during tick evolution [21]. The possible role of CLE in nutrition, osmoregulation or excretion, synthesis of amino acids, and major B vitamins has been proposed [7, 2223]. Exclusion symbiosis and acquisition of Francisella-like endosymbiont (FLE) and Rickettsia spp. in ticks could also be speculated as a more efficient alternative for synthesizing B7 and B9 vitamins [24-25] because of the reduced genome of CLE [26]. Other possible factors could be geography, lateral gene transfer, or tick feeding on a shared vertebrate host. There is a gap in our understanding of the ecological 
factors which could facilitate horizontal transfer $(\mathrm{HT})$ of endosymbionts among tick species. The examination of internal tick organs including salivary glands (SG) have indicated a high concentration of CLE $[7,18]$; therefore, feeding on a shared vertebrate host could be an important determinant for horizontal transfer $(\mathrm{HT})$.

In this study, the microbiome of the lone star tick was determined by using the 16S rRNA sequencing approach. The aims of this study were: a) to determine the composition of the microbiome across the life cycle in unfed and blood-fed immature and mature tick developmental stages, b) to assess the stability of the microbiome in unfed and blood-fed individually dissected tissues (salivary glands, midgut, and ovary), c) to assess the microbiome composition of field-collected ticks and, d) to test and validate the hypothesis of acquisition of FLE at the expense of CLE.

\section{Material And Methods}

\section{Ticks and animals:}

Laboratory-reared ticks: Laboratory-reared lone star ticks (A. americanum) used in this study were purchased from the Oklahoma State University Tick Rearing Facility. Adult ticks were infested on sheep and allowed to feed until repletion. Engorged ticks were cleaned with $70 \%$ ethanol and placed separately in sterile vials and kept in an incubator according to standard practices for maintaining sterile condition [27] at $80-90 \% \mathrm{RH}$ under a $14 / 10 \mathrm{~h}$ L/D photoperiod at $27 \pm 2^{\circ} \mathrm{C}$ until they laid eggs. Then, eggs were transferred to a sterile vial and kept in an incubator under the same conditions until they hatched. The larvae and nymphs were fed on hamster until detachment and kept in the incubator under the same conditions as mentioned above to allow them to molt into the next developmental stage (nymph and adults, respectively). Newly molted adults were then fed on a rabbit, and partially blood-fed males and females were pulled after five days for microbiome analysis. Newly molted larvae, nymphs, and adults were used in this study to ensure that bacteria were not acquired from the environment. The protocol for all animal experiments was approved by the Institutional Animal Care and Use Committee of The University of Southern Mississippi.

Field-collected ticks: Questing nymphal and adult stages of the lone star tick were collected by dragging vegetation in Delaware state parks (New Castle, Kent, and Sussex Counties) in June-July 2020 with a 1$\mathrm{m}^{2}$ white flannel drag cloth. Ticks were also collected alive into 14-mL plastic centrifuge tubes in Brown county on June-June via dragging of vegetation with $1-\mathrm{m}^{2}$ cloth drags and in DeWitt county with dry-ice baited traps. Ticks were identified alive at the INHS Medical Entomology Lab and all nymphal and adult (female and male) Am. americanum collected were shipped in secured tubes for microbiome analysis. Questing nymphs and adult lone star ticks were similarly collected from vegetations in field locations in Maryland and Illinois.

\section{Tick life stages and DNA extraction}


Five different tick generations were maintained and reared in the lab from five separate engorged females. Tick samples from different life stages, namely egg, larval, nymphal, adult, and various tissues, were collected. All samples (eggs, larvae, nymphs, and adults) were surface sterilized with $2 \%$ bleach and washed twice in $70 \%$ ethanol for $5 \mathrm{~min}$ followed by three washes in sterile deionized water to disinfect the body surface and remove external debris in preparation for DNA extraction. The samples from each developmental stage were divided into five pools of egg clutch ( 200 eggs each), thirty unfed larvae, thirty fed larvae, ten unfed, and ten fed nymphs per pool. For microbiome analysis of adult ticks, five separate unfed and partially fed males and female ticks were subjected to DNA extraction. For microbiome analysis of adult female tick tissues, DNA was extracted from dissected tissues (salivary gland, midgut, and ovary) of five separate partially fed and unfed female ticks.

The blood-fed female Am. americanum were dissected within four hours of removal from the rabbit. Ticks were dissected, and tissues were collected (salivary glands, midgut, ovary) by following standard procedure [28]. DNA was extracted using a DNeasy Blood and Tissue Kit according to the manufacturer's protocol (QIAGEN, Germantown, MD, USA). Total DNA was quantified using a Nanodrop spectrophotometer (Thermo-fisher Scientific, USA). The extraction procedures were performed in a biosafety cabinet to ensure that the samples were protected against environmental contamination.

\section{Library preparation for $16 \mathrm{~S}$ Illumina sequencing and multiplexing}

16S Metagenomic Sequencing Library Preparation protocol from Illumina was followed for library preparation and multiplexing. Dual indexes were used for library preparation. All the primers for amplicon PCR (first PCR) and indexing PCR (second PCR) were ordered from Integrated DNA Technologies (IDT, Coralville, lowa, USA). Information about all the primers used has been provided in Additional File 1. Multiplexing of tick samples was performed by targeted $16 \mathrm{~S}$ amplicon sequencing. The gene specific sequences used in this protocol target the $16 \mathrm{~S}$ V3 and V4 region. Primers for amplicon PCR were selected from the previously published study [29]. Illumina adapter overhang nucleotide sequences were added to the gene-specific sequences. The full-length primer sequences for amplicon PCR (first PCR) were 5'TCGTCGGCAGCGTCAGATGTGTATAAGAGACAGCCTACGGGNGGCWGCAG-3' (Forward), and 5'GTCTCGTGGGCTCGGAGATGTGTATAAGAGACAG GACTACHVGGGTATCTAATCC-3' (Reverse) where the forward primer overhang sequence was TCGTCGGCAGCGTCAGATGTGTATAAGAGACAG, and the reverse primer overhang sequence was GTCTCGTGGGCTCGGAGATGTGTATAAGAGACAG. The V3-V4 locus specific forward primer was CCTACGGGNGGCWGCAG and the reverse primer was

GACTACHVGGGTATCTAATCC. As mentioned above, workflow was based on the $16 \mathrm{~S}$ Metagenomic Sequencing Library Preparation protocol from Illumina (San Diego, CA, USA). To determine bias introduced by PCR amplification and sequencing error [30-31], we included a commercially available Mock Microbial Community Standard, ZymoBIOMICS (catalog\#D6306). Dual indexes (i5 and i7) were picked from Nextera Index Kit V2 (Illumina, San Diego, CA) to individually barcode the amplicons. Specific dual indexes combination for each sample was added to the index PCR (second PCR) primers. After primer designing, amplicon and index PCR primers were ordered from Integrated DNA Technologies (IDT). PCR amplifications and Illumina sequencing were performed for the mock bacterial community in the 
same manner as for tick samples. Thermocycling conditions for amplicon PCR ( $\left.1{ }^{\text {st }} \mathrm{PCR}\right)$ were $95^{\circ} \mathrm{C}$ for 3 min, followed by 25 cycles of $95^{\circ} \mathrm{C}$ for $30 \mathrm{~s}, 55^{\circ} \mathrm{C}$ for $30 \mathrm{~s}$, and $72{ }^{\circ} \mathrm{C}$ for $30 \mathrm{~s}$, and a final extension step of $72{ }^{\circ} \mathrm{C}$ for $5 \mathrm{~min}$. Amplicons were purified with AmPure XP beads (Agencourt Bioscience Corporation, Beverly, Massachusetts, USA) as instructed in the manufacturer's protocol. Purified PCR product was run on $1.5 \%$ agarose gel to certify the appropriate size ( $500 \mathrm{bps})$ of amplicon. Purified amplicons were each dissolved in $50 \mu$ l of nuclease-free water, and $5 \mu$ of each amplicon solute was transferred to a PCR tube for subsequent dual-index PCR. The index PCR assays (each $50 \mu \mathrm{l}$ ) contained $5 \mu \mathrm{l}$ each of forward and reverse Nextera Index primers (i5 and i7) and 1X KAPA HiFi HotStart ReadyMix (Kapa Biosystems, Wilmington, MA, USA). Cycling conditions were $95^{\circ} \mathrm{C}$ for 3 min followed by eight cycles of $95^{\circ} \mathrm{C}$ for $30 \mathrm{~s}$, $55^{\circ} \mathrm{C}$ for $30 \mathrm{~s}$, and $72{ }^{\circ} \mathrm{C}$ for $30 \mathrm{~s}$, and a final extension step of $72{ }^{\circ} \mathrm{C}$ for $5 \mathrm{~min}$. After the second PCR, PCR cleanup was performed with AMPure XP beads (Beckman Coulter, Brea, CA) and eluted in $25 \mu \mathrm{l}$ of TE buffer (Qiagen Cat No./ID: 19086). This TE buffer was used for all the dilution purposes throughout the process. 16S rRNA metagenomic libraries were quantified with KAPA Library Quantification Kit from Roche (catalog\# 07960255001, Kit code KK4844), and all the libraries were normalized to a concentration of $7 \mathrm{nM}$, and then $5 \mu \mathrm{l}$ of each sample was used to make a pool. All the first PCR and second PCR steps were followed as mentioned in the 16S Metagenomic Sequencing Library Preparation guide from Illumina. Samples were pooled in a single tube for sequencing at the University of Mississippi Medical Centre (UMMC) Genomics Core Facility. Three biological replicates of each of the controls including 1) DNA extraction blank, 2) buffer, 3) sterile water, 4) No template control, and 5) Positive DNA extraction control (commercially available Mock Microbial Community Standard, ZymoBIOMICS catalog \# D6306). These controls were also processed during library preparation (amplicon PCR and indexing PCR) and sequencing.

\section{Data Processing and Analysis}

Demultiplexed paired-end fastq files were provided by the University of Mississippi Medical Centre (UMMC), Jackson, Mississippi. Quantitative Insights into Microbial Ecology (QIIME 2, https://qiime2.org) was used for sequence analysis. A meta-data file and manifest file were created. The created metadata file was validated by Keemei [32]. The Qiime2 tutorial was followed to make the manifest file [33]. Manifest file maps sample identifiers to fastq.gz absolute file paths that contain sequence and quality data for the sample. The manifest file is a tab-separated text file. In brief, first column defined the sample ID, second column defined the absolute path to the paired end read (forward and reverse). Manifest file is compatible to sample-metadata; therefore, it could be reused to bootstrap sample-metadata. "Atacama soil microbiome" tutorial and "moving pictures tutorial" were followed to process the sequencing data. Briefly, Deblur [34] was used for trimming, primer sequence removal, sequence denoising, paired-end merging, filtering of chimeric sequences, singleton removal, and sequence dereplication. Minimum overlap of 40 bases was used for paired end merging. Resultant sequence sets obtained after Deblur processing were aligned by MAAFT (ver.7) [35], and then a phylogenetic tree was created by using FastTree (ver. 2.1) [36]. Silva_132_99\% Operational Taxonomic Units (OTU) database [37] was used to 
train the Naïve Bayes classifier. Raw sequences were submitted to the NCBI read under SRA database and obtained accession \# PRJNA728711 \& PRJNA751548.

Taxonomy and metadata tables were exported from QIIME2 and uploaded as input files to the Microbiome Analyst web-based interface for visualization of bacterial relative abundances and correlation network analysis as previously described [38-39]. A minimum count of 10 and minimum prevalence of $20 \%$ was applied to remove low count filter from the uploaded OTU table, and a $10 \%$ interquartile range was used as the minimum for removing low variance filter features from the OTU table. Network correlation maps were inferred based on the Sparse Correlations for Compositional data (SparCC) approach [40]. This approach uses the log-transformed values to carry out multiple iterations which subsequently identifies taxa that are outliers to the correlation parameters [38].

\section{Statistical Analysis}

In our analyses, each sample was rarefied to 2700 sequences, and the rarefied feature table was used for rarefaction analysis. Alpha and beta-diversity was measured by using different metrics. To compare significance in the bacterial diversity across different tick life stages and tissues, we explored the community richness and evenness using Faith_pd and Pielou_e/evenness metrics, respectively, as alpha diversity measures. The alpha-diversity group significance command creates boxplots of the alphadiversity values and significant differences between groups are assessed with the Kruskal-Wallis test. The beta-diversity command uses boxplots to visualize the distance between samples aggregated by groups specified in the metadata table file. Significant differences are assessed using a PERMANOVA analysis. Faith's phylogenetic diversity (faith_pd) is an unweighted measure of alpha-diversity based on phylogenetic distance in a particular sample while Pielou's evenness measures how evenly species are distributed in a sample. Kruskal-Wallis non-parametric tests $(p \leq 0.05)$ were performed to determine statistical significance of alpha-diversity metrics by using QIIME 2. Weighted and unweighted UniFrac Metrics [41] were used for beta-diversity analysis. Weighted and unweighted UniFrac metrics [41] determined the Beta-diversity of the whole tick life stage and tissue samples. EMPeror [42] was used to create the Principal Component Analysis (PCOA) plot, and PERMANOVA tests $(p \leq 0.05)$ were used to test the statistical significance of beta-diversity measurements. The bacterial community profile as a function of the beta diversity in both field-collected and lab-raised ticks was explored using the Principal component analysis (PCoA) plot.

\section{Relative quantification of CLE and FLE in field collected ticks from Delaware}

Relative expression of CLE and FLE was estimated in unfed (UF) and partially fed (PF) tick tissues (salivary gland, gut, and ovary) by using qRT-PCR (Fig. 10). Actin was used as a housekeeping gene (Additional File 2). Due to limitations of the amount of DNA in the sample, three biological replicates and two technical replicates were used during qRT-PCR assay. Briefly, 64 ng of good quality DNA (260/280

2.0) was used for each technical replicate. Primers used in this study have been provided in Table S5. The qRT-PCR reaction mixture was comprised of $350 \mathrm{nM}$ of each primer, and $\mathrm{iTaq}^{\mathrm{TM}}$ Universal SYBR ${ }^{\circledR}$ Green 
Supermix (catalog \#1725121). Reaction mixtures were subjected to $95^{\circ} \mathrm{C}$ for 6 min, followed by 45 cycles of $95^{\circ} \mathrm{C}$ for $27 \mathrm{~s}, 50^{\circ} \mathrm{C}$ for $30 \mathrm{~s}$, and $72^{\circ} \mathrm{C}$ for $35 \mathrm{~s}$ using the CFX96 Real Time System (BioRad Inc. Hercules, CA, USA).

\section{Results}

\section{Demultiplexing of sequences}

Illumina MiSeq sequencing generated a total of 6,832,744 demultiplexed paired-end sequences. Sequence counts in tick samples had an average of 88,737 sequences for each tick sample (Additional File $3 \mathrm{~A}$ ). Rarefaction curves relating sampling depth to the number of observed OTUs approached saturation plateau after a sampling depth of approximately 2700 sequences (Additional File 4A). For all 77 samples, including all biological replicates, the total number of OTUs obtained were 1666. From fieldcollected tick tissues, we generated a total of 3,972,116 raw, demultiplexed paired-end sequences had an overall average read of 30,092 sequences per tick. For all 116 field collected samples, including biological replicates, the total number of OTUs obtained was 12891 (Additional File 3B). Each biological sample was rarefied down to a sampling depth of 5000 sequences per sample (Additional File 4B). Overall, comparing the rarefaction analysis between lab-raised and field-collected tick samples showed a massive difference in the number of OTUs observed at similar sampling depth, with fewer OTUs observed in labraised ticks indicating a significant difference in diversity.

\section{Composition of the microbial communities in life stages and tick tissues from lab-raised ticks}

In whole tick samples, among all life stages, $>98 \%$ of reads were covered by Francisellaceae, Midichloriaceae, Rickettsiaceae, and Spirochaetaceae. Still, in tick tissues (salivary gland, midgut, ovary), $>98 \%$ reads are covered by Francisellaceae, Midichloriaceae, Rickettsiaceae, Spirochaetaceae, Coxiellaceae, Caulobacteraceae, and Rhizobiaceae. In unfed and blood-fed life stages (Fig. 2A) and tissues (Fig. 2B), Francisellaceae was found as the most dominant family with a coverage of $\sim 70-75 \%$ reads except unfed ovary (UF-OV) in which Coxiellaceae is the most dominant bacterial family. Of the four biological replicates of unfed-ovary, Coxiellaceae (55-72\%) was the dominant family in three, while the family Francisellaceae (71\%) was abundant in one. The family Spirochaetaceae (7-18\%), Caulobacteriaceae (4-18\%), Rickettsiaceae (6\%), Rhizobiaecae ( 4\%) and Francisellaceae (10\%) were also detected in Coxiellaceae positive unfed ovarian tissues. Caulobacteraceae and Rhizobiaceae are present only in the unfed salivary gland (UF-SG) and unfed ovary (UF-OV). In UF-SG, the abundances of Caulobacteraceae and Rhizobiaceae are $5.32 \%$ and $1.81 \%$, respectively. In tick life stages, bacterial profiling is stable. In all unfed and blood-fed tick life stages, the abundance of Francisellaceae varies from $70.5 \%$ to $73.5 \%$, Midichloriaceae from 16.3 to $17.7 \%$, Rickettsiaceae from 7.5 to $9.3 \%$, and Spirochaetaceae from 1.08 to $1.58 \%$. This result suggests hardly any impact of tick-blood feeding and tick-sex (male, female) on bacterial profiling at the genus level. Bacterial families present at $<1 \%$, were categorized as others.

\section{Composition of the microbial communities from field-collected ticks}


We observed a similar pattern regarding bacterial taxonomy's presence and relative abundances within tick tissues from Illinois, Delaware, and Maryland. The bacterial proportion was cataloged from the phylum down to the species level. Due to the limitation of our classifier to completely resolve identification at the genus or species level, family level classification was used to define the patterns of bacterial assemblages identified from the field-collected ticks. The family Francisellaceae (12\%-55\%) was the dominant bacterial family regardless of tick tissues and geography. The only exception was the presence of Coxiellaceae $(<50 \%)$ in a replicate of unfed salivary gland (UFSG) and Rickettsiaceae ( $>70 \%)$ in a replicate of partially-fed salivary gland (PF-SG) from Illinois (Fig. 3A), and Rickettsiaceae (100\%) in a replicate of the unfed midgut (UF-MG) from Delaware (Fig. 3B). Other predominantly identified bacterial family includes Nocardiaceae (>1\%-20\%), Rickettsiaceae (10\%-100\%), and Rickettsiales (3\%-21\%) (Fig. $3 A-C)$. An interesting observation was the presence of the family Coxiellaceae in partially fed salivary glands (PF-SG; 6\%-17\%) and partially fed midgut (PF MG; 15\%-18\%) from Delaware ticks (Fig. 3B). The relative profiles of those taxa that were completely resolved to the genus and species level is attached to the supplementary data (Additional File 5).

\section{Diversity analysis}

\section{Lab-raised ticks}

The Alpha diversity comparison across different isolated tissues from lab-raised tick colonies showed significant differences in microbial richness Kruskal-Wallis; $\mathrm{H}=15.87$; $\mathrm{p}$-value $=0.026$ ) and evenness (Kruskal-Wallis; $\mathrm{H}=17.63$; $\mathrm{p}$-value $=0.014$ ) with no observed differences across developmental stages. Across different life stages from lab-raised tick colonies, unfed nymphs (Faith_pd value $=1.861961$ ) and fed males (Faith_pd value $=1.40254$ ) had the highest and lowest diversity richness index, respectively. In contrast, fed larvae (Pielou_e/evenness $=0.645623$ ) and fed males (Pielou_e/evenness $=0.587806$ ) showed the highest and lowest diversity evenness metric (Fig. 4A). Amongst isolated tissues, unfed midgut (Faith_pd value $=1.667971)$ and partially fed midgut (Faith_pd value $=1.375176)$ had the highest and lowest diversity richness index, respectively. In contrast, unfed ovary (Pielou_e/evenness $=0.6872987$ ) and partially fed salivary gland (Pielou_e/evenness $=0.5937431$ ) showed the highest and lowest diversity evenness metric (Fig. 4A). Alpha diversity was significantly higher in unfed ovaries when compared with fed ovaries (Pielou_e/evenness Kruskal-Wallis; $\mathrm{H}=3.84$; $\mathrm{p}$-value $=0.05$; $\mathrm{q}$-value $=0.127$ ) as represented in Additional File 6, while unfed midgut showed a significantly higher alpha diversity compared to fed midgut tissues based on the Faith_pd measure of microbial richness (Faith_pd KruskalWallis; $\mathrm{H}=5.77$; $\mathrm{p}$-value=0.016; q-value $=0.19$ ) as shown in figure 4A (Complete list in Additional File 7).

Field-collected ticks

The Alpha diversity comparison between unfed and partially fed tissues from field-collected ticks showed significant differences in richness (Kruskal-Wallis; $\mathrm{H}=53.33$; $\mathrm{p}$-value $=0.005$ ) and evenness (Kruskal-Wallis; $\mathrm{H}=50.76$; $p$-value=0.001). Pairwise comparison of alpha diversity between unfed and partially-fed tissues showed a significant difference between the unfed and partially fed ovarian tissues isolated from ticks from Maryland (Faith_pd Kruskal-Wallis; $\mathrm{H}=3.86$; $\mathrm{p}$-value=0.05; q-value=0.200. Pielou_e/evenness; 
$\mathrm{H}=3.86 ; \mathrm{p}$-value $=0.05 ; \mathrm{q}$-value $=0.27$ ) and Delaware (Faith_pd Kruskal-Wallis; $\mathrm{H}=7.68 ; \mathrm{p}$-value $=0.007$; qvalue $=0.12$. Pielou_e/evenness; $\mathrm{H}=9 ; \mathrm{p}$-value $=0.003$; $q$-value $=0.21$ ). The complete list of pairwise analysis is included as Additional File 8 and 9). There were no observed significant differences from unfed and partially fed tissue isolated from ticks collected from Illinois (Fig. 4B).

The PCoA of the weighted (Axis $1 ; 85.33 \%$ and Axis 2; $13.90 \%$ ) and unweighted (Axis $1 ; 17.10 \%$ and Axis $2 ; 14.64 \%$ ) UniFrac distances in unfed and fed life-stages of lab-raised ticks revealed no unique clustering pattern in the bacterial communities (Fig. 5A and B respectively). Upon pairwise comparison, PERMANOVA (Permutational multiple analysis of variance) indicated that few pairs of tick life-stage samples contained statistically different bacterial communities to each other, such as unfed-nymph and fed-male $(p=0.026)$, or unfed-nymph, and unfed-larvae $(p=0.007)$ (Complete similarity comparison is in

Additional File 10). When community profiles and similarities were explored for isolated tissues from labraised ticks, the weighted UniFrac distance matrix revealed that the unfed ovary (UF-OV) was distantly clustered compared to other tissue samples suggesting that it contains a unique dominant bacterial community than all other tissue samples (Fig. 5C).

In contrast, the unweighted UniFrac distance matrix revealed no unique clustering pattern (Fig. 5D). The unique clustering pattern presented by the unfed ovary tissues in Figure $6 \mathrm{C}$ was further supported by the relative abundances of the bacterial families identified in this particular tissue (Fig. 2B) and the Bray_Curtis pairwise comparison which showed a significant difference between the composition of unfed ovary tissues and fed midgut tissues (Additional File 11). The dominant bacterial family in the unfed ovary was Coxiellaceae ( 37\%) which, is absent in all other tissue samples. In all other tissue samples, commonly dominant bacterial families were Francisellaceae, Midichloriaceae, Rickettsiaceae, and Spirochaetaceae, and they covered more than $90 \%$ of reads. There was no observed distinct clustering pattern in the community profiles of tick tissues isolated from field-collected ticks using both the weighted and unweighted UniFrac distance matrixes (Fig. 5E and F). The dataset showing the pairwise comparison in field-collected ticks based on the weighted and unweighted UniFrac metrics is detailed in Additional Files 12 and 13.

\section{Network correlations in the microbiome of lab-raised ticks}

A correlation network generated using the SparCC algorithm identified 10 OTUs at the family level with 14 significant correlations across the different life-stages from ticks raised in the lab (Fig. 6A), of which seven were positively correlated. Of the ten identified OTUs, $80 \%$ were not fully represented in all our samples. Only those belonging to the families Rickettsiaceae and Midichloriaceae were observed to be fully detected in all tick life-stages. Interestingly these two families (Rickettsiaceae and Midichloriaceae) were positively correlated with a significantly high correlation threshold of 0.9963 (Fig. 6A).

Rickettsiaceae and Midichloriaceae had consistently higher dominance across the tick life stages (Fig. $6 \mathrm{~B}-\mathrm{C})$.

A total of 12 OTUs with 29 significant correlations were identified when the SparCC correlation algorithm was applied to samples representing tissues from lab-raised tick colonies. Approximately $48 \%$ of these 
correlations were positively correlated amongst different bacterial families, notably Francisellaceae, Rickettsiaceae, and Midichloriaceae, all of which were equally represented in all sampled tick tissues (Fig. 7A). Coxiellaceae was dominant in unfed ovarian tissues of lab-raised tick colonies (Fig. 2A and 7B) and, was positively correlated with bacteria in the families Xanthobacteraceae and Rhizobiaceae, both of which showed strong negative correlation to the families Francisellaceae, Midichloriaceae, and Rickettsiaceae (Fig. 7A) which were exclusively enriched in all other tissues except for the unfed ovaries (Fig. 7C-E). Similarly, the unfed ovarian tissue exclusive Coxiellaceae was also identified to positively correlate to an unknown bacterial taxon that exhibited positive correlations to the families Francisellaceae, Rickettsiaceae, and Midichloriaceae (Fig. 7A).

\section{Network correlations in the microbiome of field-collected tick colonies}

Our correlation analysis of field-collected tick samples only showed significant correlations within samples collected from Illinois and Delaware. We identified 12 significant correlations among nine bacterial families from ticks collected in Illinois, nine of which were positive correlations (Fig. 8A). A negative correlation existed between the family Coxiellaceae enriched in unfed salivary gland tissues (Fig. 8B), and Francisellaceae, which was present across all samples (Fig. 8C). Only four bacterial families were significantly correlated among ticks collected in Delaware (Fig. 9A-D), and all were negative correlations.

\section{Relative quantification of FLE and CLE in field-collected tick tissues}

Relative quantification of FLE was found higher than CLE in tick tissues from field-collected $\mathrm{Am}$. Americanum from Delaware (Fig. 10). The relative load of FLE were found to be higher in tick tissues except for unfed ovary in which FLE and CLE levels are nearly equal (Fig. 10). Compared to 16S data, interestingly, our QPCR data detected the expression of CLE in unfed salivary glands, unfed midgut, and partially fed ovarian tissues where as they were not detected in the $16 \mathrm{~S}$ data. The relative load of FLE was higher in all fed tissues compared to unfed tissues with only fed midgut showing significant differences (Fig. 10).

\section{Discussion}

The consistent occurrence of Francisella in both our lab-grown and field-collected tick samples suggests its systemic association with ticks. Our analysis has assigned abundant sequences to Francisella (7075\%), followed by Midichloria (14-17\%) and Rickettsia (6-9\%), excluding unfed ovary tissue. The richness of Francisella in immature and mature life developmental stages and individually-dissected tissues (except for the unfed ovary) supports the notion that it sustains an obligatory association with all feeding stages and outcompetes other bacteria. The relative abundance of Midichloria and Rickettsia was considerably lower than Francisella, signifying their link with the tick as facultative endosymbionts. However, competitive interactions among endosymbionts, pathogenic microbes, have been shown to increase virulence of pathogenic microbes, and complications in vertical transmission were suggested to influence obligatory or facultative endosymbionts in ticks [43]. Consistent dominance of Francisella in 
tick life stages and tissues suggests its significance in Am. americanum development and other biological processes. Perhaps the Am. americanum ticks are sheltering Francisella by creating a favorable niche within various tissues vector for the colonization, propagation, and trafficking of Francisella at the expense of other endosymbionts and microbial communities. The tick vector sequestered vital nutrients from Francisella for development, and in return provided the niche for Francisella to survive and thrive within the tick vector. Synergistic and antagonistic interactions among pathogenic and non-pathogenic rickettsial species could also play an important role in the prevalence of microbes within the tick vector. The interspecies competition among Midichloria and Rickettsial species could also cause their decreased prevalence within the arthropod vector. For example, dominant Rickettsia peacockii blocked vertical transmission of $R$. rickettsii in the ovary of Dermacentor andersoni [44-45]). The dominance of Francisella-like endosymbionts (FLEs) in Am. americanum might be location-specific, as demonstrated for Coxiella and Rickettsia [46-47], and of fundamental importance because of its occurrence in several other tick species such as Dermacentor occidentalis Marx, 1892, Dermacentor albipictus (Packard), Dermacentor nitens Neumann, 1897, D. andersoni Stiles, 1908, Dermacentor hunteri Bishopp, 1912, and Dermacentor variabilis (Say, 1821) [45-49]. Still, the nature of their symbiotic relationship is yet to be explained. In this study, field-collected tick tissues from Illinois, Delaware, and Maryland also demonstrated the presence and dominance of FLEs, suggesting their establishment in field populations of Am. americanum ticks, although their presence seems much prominent in lab-grown colonies (Fig. 10).

We found differences in the diversity metrics between lab-raised and field-collected ticks colonies. The observed_OTUs metrics, a quantitative measure of the total number of bacteria, showed that fieldcollected ticks have about 100 folds more bacteria than lab-raised ticks irrespective of sample type (Fig. S1). We also found that field-collected ticks have a higher diverse microbiome regardless of the tick location than lab-raised tick colonies. To further strengthen this observation, despite the fact Francisellaceae was the dominant bacterial family in both tick groups, field-collected ticks also harbored other bacterial families at a relatively high abundance contributing to a more diverse microbiome. This finding implies that the tick environment plays a significant role in enriching certain bacteria members of the tick microbiome. The lab-raised ticks have been maintained under strict laboratory conditions with limited vertebrate hosts and have less richness in their microbial communities. It seems possible that the differences in the diversity observed amongst life stages of field-collected and lab-raised ticks is due solely to the continuous exposure to environmental bacteria encountered by questing ticks in the field. A closer look at bacterial profile in Figs. 2 and 3 clearly indicates that with the exception of the presence of Francisellaceae in both laborotory raised and field-collected ticks, several bacterial family were exclusively detected in field-collected ticks such as Propionibacteriaceae, Nocardiaceae, Dermabacteraceae, Rickettsiales and Staphylococcaceae all of which are known to be environmental residents in soils and are predominant on both human and animal skin. These findings provide further support for the hypothesis that the tick microbiome may play a contributing factor to the incidence of the alpha-gal phenomenon across different region in the United States. 
An earlier study conducted on field-collected Am. americanum ticks from Indiana showed $\sim 40 \%$ of Coxiella-like endosymbionts, followed by 5\% rickettsial endosymbionts [46]. However, ticks collected from North Carolina harbored a preponderance of Rickettsia-like endosymbionts over Coxiella [47]. In the current study, the most abundant bacterial family is Francisella at the organismal level in both immature and adult tick life stages and individually dissected tissues (excluding unfed ovary), suggesting that the microbiome composition of this tick varies with geographical distribution. The presence of Coxiella in unfed ovary tissue indicates its obligate symbiont nature, and another study has shown its presence in the ovary (to show its maternal transmission) and Malpighian tubules to suggest its possible role in osmoregulation and excretion [7, 22-23]. Surprisingly, CLE was neither detected in tick developmental life stages nor tissue samples, except in the unfed ovary. CLE, an obligatory endosymbiont, is required for tick survival and reproductive fitness [50]. CLE has been implicated for its possible role in synthesizing amino acids and several $B$ vitamins [7, 22-23]. Possible reasons for the replacement of CLE in Am. americanum could be the acquisition of FLE and rickettsiae established as alternative obligate symbionts in some tick species [25]. All genes required for vitamin B9 and B7 biosynthesis are also present in Rickettsia and FLE endosymbionts, respectively. In instances where multiple endosymbionts provide a similar advantage to the host, the presence and maintenance of all endosymbionts are not expected; it does not add additional help to the tick host [51].

It was a common understanding that hematophagous niches are commonly driven by evolutionarily stable symbiotic interactions in several arthropods [52-53]. Contrary to that, an elegant study proposed that obligate symbioses are relatively unstable in obligate hematophagous ticks [25]. In that study, six genera of distinct endosymbionts were present in the castor bean tick (Ixodes ricinus) and the African blue tick (Rhipicephalus decoloratus) but no symbiotic community structure was found fixed and stable across the tick phylogeny [25]. The success of horizontal and vertical transmission patterns in ticks could also modify symbiotic interactions [25]. This fact could also explain why there is low evolutionary stability of the symbiosis between Am. americanum ticks and CLE or CLEs are missing from most of our tick samples in the current study.

CLE follows two different evolutionary strategies. Some CLEs are highly specialized to the tick host from ancient times, followed by co-diversification. For example, the Rhipicephalus genus and CLE lineages have co-diversified together. The emergence of the Rhipicephalus genus and original CLE infection cooccurred, 14 million years ago $[25,54]$. On the other hand, other CLEs appear to be acquired through horizontal transfer (HT) from unrelated or accidental host species. Such a pattern has also been observed in other endosymbionts such as Wolbachia, which occurs frequently in insects, similar to CLE in ticks. A recent study also supports the hypothesis of frequent replacement of obligate symbiont CLE in ticks [25]. Our data provide strong evidence that CLE replacement by FLE occurs in Am. americanum populations; otherwise, FLEs are rare in ticks and not frequently found in arthropods [25-26, 45, 49, 55-56]

Earlier studies proposed that among Amblyomma tick species, CLEs have reduced genomes, a feature associated with microbes that are long-term or early evolved endosymbionts [57]. A wide distribution of genetically differentiated strains of CLE across the tick phylogeny also specifies its ancient symbiotic 
relation with ticks [25]. Unlike the highly reduced genome of CLEs, FLEs have minimal genome reduction and evolved recently from a mammalian pathogen Francisella tularensis [26] and have more superior biosynthetic metabolic capabilities than CLEs. Therefore, it is highly likely that it has replaced ancestral CLEs with reduced functionality in Am. americanum developmental stages and tissue samples, as evident from the data presented here. A recent study has also suggested replacement of endosymbiont CLEs with FLEs in another Amblyomma tick species, the Gulf Coast tick (Amblyomma maculatum), for the same reason [26]. It is also possible that $A m$. americanum replaced CLE at the expense of functionally important symbiotic genes (via lateral gene transfer), with FLEs which are functionally more efficient than CLEs because CLE has a reduced genome and is less efficient in metabolic and biosynthetic capabilities, including that of B vitamin synthesis. Perhaps, this is the key evolutionary mechanism of how ticks retain their capacity to synthesize vitamin B even without containing endosymbionts.

Previously, this pattern has been reported from some filarial nematodes, which demonstrated the ability to live and reproduce without obligate symbionts through lateral gene transfer [58]. A close examination of Ixodes scapularis, a tick species deficient in CLE, did not show any sign or evidence of lateral gene transfer [59]. However, it contained a rickettsial endosymbiont, Rickettsia buchneri, which could synthesize the B9 vitamin [24]. The presence of Rickettsia buchneri provides a possible explanation for both the absence of CLE and no evidence of lateral gene transfer. An unexplored ecological pathway area facilitating the horizontal transfer (HT) of endosymbionts among tick species needs to be thoroughly investigated. Co-feeding of different tick species on a shared vertebrate host could be an important determinant. Reports have also shown that the salivary glands of blood-fed ticks contain high levels of CLE in some tick species $[7,18]$. Thus, the co-feeding of tick species on a shared vertebrate host could serve as an ecological arena for exchanging endosymbionts. Earlier studies have suggested that tick pathogens also colonize with closely related endosymbionts, and these endosymbionts also appear to promote closely related pathogen acquisition and transmission [60]. The FLEs are known to have evolved from the pathogen Francisella tularensis $(\mathrm{Ft})$; however, unlike virulent $\mathrm{Ft}$, its transmission and virulence in humans are enigmas [61-62]. A wide distribution of FLEs in other tick species has been further highlighted by the reports such as $>94 \%$ of FLE positives among $D$. variabilis, $D$. andersoni and $D$. occidentalis ticks in the western United States [45, 63], 41\% FLE-positives in D. occidentalis ticks from California (Western United States) but without Ft infection [64]. In another earlier study, D. andersoni ticks collected from Oregon and Montana (northwestern United States) showed FLE and Ft accounted for $80 \%$ $(60 \% \mathrm{FLEs}$ and $20 \% \mathrm{Ft}$ ) of midgut microbiome [65], suggesting that genetic similarity of FLE and $\mathrm{Ft}$, and geography are both probably contributing to inflating Ft infection rates. More studies are needed to clarify the involvement of FLEs in Ft infection and how factors such as geography and genetic similarity are involved.

Our network analysis showed a strong positive correlation between Rickettsiaceae and Midichloriaceae families in lab-raised tick life-stages and between Francisellaceae, Rickettsiaceae, and Midichloriaceae in tissues isolated from lab-raised ticks. Interestingly, the presence of the Rickettsiaceae family was shown to be positively correlated to Midichloriaceae across different life-stages of lab-raised tick colonies, indicative of a potential synergistic relationship between bacteria belonging from these two families. This 
observation was also reported in another study [16]. It showed the Rickettsia parkeri colonization of the tick tissue facilitates replication of the endosymbiont Candidatus Midichloria mitochondrii (CMM) in Am. maculatum ticks. Lejal et al. [66] also identified a substantial prevalence of CMM in Ixodes ricinus ticks that were positive with bacteria in the Rickettsia genus.

The Coxiellaceae is replaced with the Francisellaceae in Am. americanum ticks and this pattern was further strengthened by analyzing the correlations between bacterial families identified in tissues of labraised tick colonies. While we observed no direct correlation among bacteria in the Coxiellaceae family and Rickettsiaceae, Francisellaceae, and Midichloriaceae, the Coxiellaceae family was positively correlated with Rhizobiaceae and Xanthobacteraceae, both of which were negatively correlated to Rickettsiaceae, Francisellaceae, and Midichloriaceae bacterial families. Both Rhizobiaceae and Xanthobacteraceae are non-resident, opportunistic bacteria that ticks acquire from their environment [17, 46-67]. Lejal and colleagues [66] detected a significant abundance of a Rhizobiaceae-Multi_1 in Rickettsia-positive Ixodes ricinus ticks, suggesting a completely different observation of our findings that Rhizobiaceae was negatively correlated with the Rickettsiaceae, but positively correlated with Coxiellaceae. This finding could present a potential interaction between known bacterial endosymbionts and possible environmental bacteria transiently acquired by ticks. An understanding of how these environmental microbes change the dynamics of the tick microbiome requires further attention.

The presence of specific microbes in tick salivary glands is vital in the context of a-gal syndrome (AGS) as tick bites are believed to be responsible for causing AGS in humans. The lone star tick possesses a-gal antigens in its salivary gland and saliva which are hypothesized to be prime culprits of AGS [1]. The gene that codes for the enzyme a-1,3-galactosyltransferase, has not been identified in a tick so far, indicating that the microbiome of the tick salivary gland could be one major contributor of a-gal antigens [68]. Several pieces of evidence support this hypothesis. One important tick-borne bacterial pathogen, Anaplasma phagocytophilum, is linked with an increase of alpha-gal antigen [69]. The recent change of guard within Am. americanum (CLE replaced by FLE) points to the potential role of the tick microbiome in the emergence of AGS. The exclusive replacement of the Coxiellaceae with Francisellaceae in both labraised and field-collected Am. americanum ticks could shed more light on the role played by this tick in the emergence of AGS. While there are no current studies on when the pathogenic Francisella tularensis switches to an endosymbiont, Gerhart et al [25] argued that an FLE of Am. maculatum recently evolved to becoming an endosymbiont. Similarly, it is worth noting that the emergence of AGS in humans and the incrimination of Am. americanum in inducing this condition are recent development. Understanding whether these two phenomena took place concurrently or simultaneously will significantly fill a huge gap in our understanding of the microbiome-tick vector interaction in the context of AGS.

An elegant recent study conducted on the human gut microbiome reported gut resident microbes from the Enterobacteriaceae family, including Escherichia coli, Pasteurellaceae genera, Haemophilus influenza, and Lactobacillus species, can exhibit a 1,3-galactosyltransferase activity, which indicates that the presence of an enzyme in these microbes could contribute to the devolvement of an a-gal antigen [70]. This study also identified specific genes exhibiting a-1, 3-galactosyltransferase bacterial sequences in 
their shotgun sequencing data [70]. An earlier study reported microbes from Rhizobiaceae and Caulobacteriaceae families possessing novel lipid A a- $(1,1)$-GalA transferase gene(rgtF) [71]. This enzyme could also be a potential source of an a-gal antigen. In this study, we identified microbes from Rhizobiaceae and Caulobacteriaceae family in Am. americanum salivary glands samples. The microbes from these families could contribute to the synthesis of an a-gal antigen or overall a-gal signature in tick salivary glands. The tick microbiome possessing an uncharacterized enzyme with a glycoside hydrolase, glycosyltransferase, or similar function as a-1, 3-galactosyltransferase, is yet to be investigated.

\section{Conclusion}

In this study, a detailed and comprehensive 16S rRNA sequencing revealed a stable microbiome composition in all developmental stages of the lone star tick (Am. americanum) and the traditionally associated CLE was absent in these developmental stages (excluding the unfed ovary), thus pointing to a solid niche for vertical transmission to the next generation. Interestingly, the Am. americanum ticks investigated appear to have acquired another tick endosymbiont, FLE, at the expense of CLE. Levels of FLE were significantly higher in lab-maintained ticks than field-collected ticks, possibly due to exposure to limited host species used for tick rearing compared to more diverse vertebrate hosts in the wild, and/or controlled lab conditions compared to field environmental conditions. Historically, CLE is known to benefit its tick host, synthesizing vitamin B and co-factors that aid in osmoregulation, excretion, and reproduction. The possible rationale for acquiring and establishing FLE at the expense of CLE could be the reduced genome of CLE leading to impaired vitamin B synthesis when compared to FLE. FLE coverage in tick life stages was $\sim 70 \%$ of total reads, suggesting its significance in tick physiology. An inverse relationship between the number of FLEs and SFGR has been demonstrated in Dermacentor occidentalis suggesting that FLEs can interfere with Spotted Fever Group Rickettsia (SFGR) colonization. It has also been shown that a high abundance of FLEs is positively correlated with the acquisition of pathogen Francisella novicida. Could this positive correlation between FLE and pathogen Francisella novicida lead to a possible infection of Francisella novicida in lone star ticks in the east-central United States soon?

\section{Declarations}

\section{Acknowledgements}

This article reports the results of research only. Mention of a proprietary product does not constitute an endorsement or a recommendation by the USDA for its use. The USDA is an equal opportunity provider and employer.

\section{Funding}


This research was principally supported by USDA NIFA award \# 2017-67017-26171, Pakistan-US Science and Technology Cooperation award (US Department of State); the Mississippi INBRE (an institutional Award (IDeA) from the National Institute of General Medical Sciences of the National Institutes of Health under award P20GM103476). The funders played no role in the study design, data collection and analysis, decision to publish, or preparation of the manuscript.

\section{Author Information}

School of Biological, Environmental, and Earth Sciences, University of Southern Mississippi, Hattiesburg, MS 39406, USA

Deepak Kumar, Surendra Raj Sharma, Abdulsalam Adegoke, Shahid Karim

Delaware Division of Fish \& Wildlife, Delaware Mosquito Control Sect. 2430 Old County Rd. Newark, DE 19702, USA

Ashley Kenndey

Illinois Natural History Survey, University of Illinois at Urbana-Champaign, Champaign, Illinois, 61820, USA

Holly C. Tuten

Invasive Insect Biocontrol \& Behavior Laboratory, USDA ARS, Beltsville, MD 20705

Andrew Y. Li

Center for Molecular and Cellular Biosciences, University of Southern Mississippi, Hattiesburg, MS 39406, USA

Shahid Karim

\section{Abbreviations}

Am. americanum: Amblyomma americanum

PCA: Principal component analysis

OUT: Operational taxanomic unit

TBD: Tick-borne disease 
TBP: Tick-borne pathogens

FLE: Francisella-like endosymbiont

CLE: Coxiella-like endosymbiont

AGS: Alpha-Gal Syndrome

QIIME2: Quantitative Insights into Microbial Ecology 2

PERMANOVA: Permutational multiple analysis of variance

SparCC: Sparse Correlations for Compositional data

\section{Ethical Approval and Consent to participate}

Not applicable.

\section{Consent for publication}

Not applicable

\section{Availability of data and materials}

Raw sequences for this study were submitted to the NCBI read under SRA database and obtained accession \# PRJNA72871154625 \& PRJNA751548.

\section{Competing interests}

The authors declare that they have no competing interests.

\section{Author contributions}

Conceptualization: Shahid Karim

Data curation: Deepak Kumar, Surendra Raj Sharma, Abdulsalam Adegoke, Shahid Karim

Formal analysis: Deepak Kumar, Surendra Raj Sharma, Abdulsalam Adegoke, Shahid Karim 
Funding acquisition: Shahid Karim

Investigation: Surendra Sharma, Deepak Kumar, Abdulsalam Adegoke, Shahid Karim

Methodology: Surendra Raj Sharma, Deepak Kumar; Abdulsalam Adegoke, Ashley Kennedy, Holly C. Tuten, Andrew Y. Li, Shahid Karim

Project administration: Shahid Karim

Resources: Shahid Karim

Supervision; Shahid Karim

Validation: Deepak Kumar, Surendra Raj Sharma, Shahid Karim

Visualization: Surendra Sharma, Deepak Kumar, Abdulsalam Adegoke, Shahid Karim

Writing, original draft: Deepak Kumar; Surendra Raj Sharma, Abdulsalam Adegoke, Shahid Karim

Writing, review \& editing: Deepak Kumar, Surendra Sharma, Abdulsalam Adegoke, Ashley Kennedy, Holly C. Tuten, Andrew Y. Li, Shahid Karim

\section{References}

1. Crispell G, Commins SP, Archer-Hartman SA, Choudhary S, Dharmarajan G, Azadi P, Karim S. Discovery of alpha-gal-containing antigens in North American tick species believed to induce red meat allergy. Front Immunol. 2019;10:1056. doi:10.3389/fimmu.2019.01056. PMID: 31156631; PMCID: PMC6533943.

2. Williams-Newkirk AJ, Rowe LA, Mixson-Hayden TR, Dasch GA. Characterization of the bacterial communities of life stages of free living lone star ticks (Amblyomma americanum). PloS one. 2014;9(7):e102130. pmid:25054227.

3. Menchaca AC, Visi DK, Strey OF, Teel PD, Kalinowski K, Allen MS, et al. Preliminary assessment of microbiome changes following blood-feeding and survivorship in the Amblyomma americanum nymph-to-adult transition using semiconductor sequencing. PloS one. 2013;8(6):e67129. pmid:23826210.

4. Fryxell RT, DeBruyn JM. The microbiome of Ehrlichia-infected and uninfected lone star ticks (Amblyomma americanum). PLoS One. 2016;11(1):e0146651. pmid:26751816.

5. Brinkerhoff RJ, Clark C, Ocasio K, Gauthier DT, Hynes WL. Factors affecting the microbiome of Ixodes scapularis and Amblyomma americanum. PLoS One. 2020;15(5):e0232398. doi:10.1371/journal.pone.0232398. PMID: 32413031; PMCID: PMC7228056.

6. Burgdorfer W, Barbour AG, Hayes SF, Péter O, Aeschlimann A. Erythema chronicum migrans-a tickborne spirochetosis. Acta Trop. 1983;40(1):79-83. PMID: 6134457. 
7. Klyachko O, Stein BD, Grindle N, Clay K, Fuqua C. Localization and visualization of a Coxiella-type symbiont within the lone star tick, Amblyomma americanum. Appl Environ Microbiol. 2007;73(20):6584-94. doi:10.1128/AEM.00537-07. Epub 2007 Aug 24. PMID: 17720830; PMCID: PMC2075054.

8. Budachetri K, Browning RE, Adamson SW, Dowd SE, Chao CC, Ching WM, Karim S. An insight into the microbiome of the Amblyomma maculatum (Acari: Ixodidae). J Med Entomol. 2014;51(1):119-29. doi:10.1603/me12223. PMID: 24605461; PMCID: PMC3956751.

9. Budachetri K, Gaillard D, Williams J, Mukherjee N, Karim S. A snapshot of the microbiome of Amblyomma tuberculatum ticks infesting the gopher tortoise, an endangered species. Ticks Tick Borne Dis. 2016;7(6):1225-9. doi:10.1016/j.ttbdis.2016.07.010. Epub 2016 Jul 20. PMID: 27460902; PMCID: PMC5048529.

10. Budachetri K, Williams J, Mukherjee N, Sellers M, Moore F, Karim S. The microbiome of neotropical ticks parasitizing on passerine migratory birds. Ticks Tick Borne Dis. 2017;8(1):170-3. doi:10.1016/j.ttbdis.2016.10.014. Epub 2016 Oct 28. PMID: 27802919; PMCID: PMC5472101.

11. Karim S, Budachetri K, Mukherjee N, Williams J, Kausar A, Hassan MJ, et al. A study of ticks and tickborne livestock pathogens in Pakistan. PLoS Negl Trop Dis. 2017;11(6):e0005681. doi:10.1371/journal.pntd.0005681. PMID: 28650978; PMCID: PMC5501686.

12. Adegoke A, Kumar D, Bobo C, Rashid MI, Durrani AZ, Sajid MS, Karim S. Tick-borne pathogens shape the native microbiome within tick vectors. Microorganisms. 2020;8(9):1299. doi:10.3390/microorganisms8091299. PMID: 32854447; PMCID: PMC7563471.

13. Narasimhan S, Fikrig E. Tick microbiome: the force within. Trends Parasitol. 2015;31(7):315-23. doi:10.1016/j.pt.2015.03.010. Epub 2015 Apr 27. PMID: 25936226; PMCID: PMC4492851.

14. Narasimhan S, Rajeevan N, Liu L, Zhao YO, Heisig J, Pan J, et al. Gut microbiota of the tick vector Ixodes scapularis modulate colonization of the Lyme disease spirochete. Cell Host Microbe. 2014;15(1):58-71. doi: 10.1016/j.chom.2013.12.001. PMID: 24439898; PMCID: PMC3905459.

15. Cirimotich CM, Ramirez JL, Dimopoulos G. Native microbiota shape insect vector competence for human pathogens. Cell Host Microbe. 2011;10(4):307-10. doi:10.1016/j.chom.2011.09.006. PMID: 22018231; PMCID: PMC3462649.

16. Budachetri K, Kumar D, Crispell G, Beck C, Dasch G, Karim S. The tick endosymbiont Candidatus Midichloria mitochondrii and selenoproteins are essential for the growth of Rickettsia parkeri in the Gulf Coast tick vector. Microbiome. 2018;6(1):141. doi:10.1186/s40168-018-0524-2. PMID: $30103809 ;$ PMCID: PMC6090677.

17. Andreotti R, Pérez de León AA, Dowd SE, Guerrero FD, Bendele KG, Scoles GA. Assessment of bacterial diversity in the cattle tick Rhipicephalus (Boophilus) microplus through tag-encoded pyrosequencing. BMC Microbiol. 2011;11:6.

18. Qiu Y, Nakao R, Ohnuma A, Kawamori F, Sugimoto C. Microbial population analysis of the salivary glands of ticks; a possible strategy for the surveillance of bacterial pathogens. PLoS One. 2014;9(8):e103961. doi:10.1371/journal.pone.0103961. PMID: 25089898; PMCID: PMC4121176. 
19. Schabereiter-Gurtner C, Lubitz W, Rölleke S. Application of broad-range 16S rRNA PCR amplification and DGGE fingerprinting for detection of tick-infecting bacteria. J Microbiol Meth. 2003;52:251-60. [PubMed: 12459246].

20. Duron O, Jourdain E, McCoy KD. Diversity and global distribution of the Coxiella intracellular bacterium in seabird ticks. Ticks Tick Borne Dis. 2014;5(5):557-63. doi:10.1016/j.ttbdis.2014.04.003. Epub 2014 Jun 7. PMID: 24915875.

21. Duron O, Noël V, McCoy KD, Bonazzi M, Sidi-Boumedine K, Morel O, et al. The recent evolution of a maternally-inherited endosymbiont of ticks led to the emergence of the Q fever pathogen, Coxiella burnetii. PLoS Pathog. 2015;15(5):e1004892. doi:10.1371/journal.ppat.1004892. PMID: 25978383; PMCID: PMC4433120.11).

22. Machado-Ferreira E, Dietrich G, Hojgaard A, Levin M, Piesman J, Zeidner NS, Soares CA. Coxiella symbionts in the Cayenne tick Amblyomma cajennense. Microb Ecol. 2011;62(1):134-42. doi:10.1007/s00248-011-9868-x. Epub 2011 May 25. PMID: 21611689.

23. Lalzar I, Friedmann Y, Gottlieb Y. Tissue tropism and vertical transmission of Coxiella in Rhipicephalus sanguineus and Rhipicephalus turanicus ticks. Environ Microbiol. 2014;16(12):365768. doi:10.1111/1462-2920.12455. Epub 2014 Apr 18. PMID: 24650112.

24. Hunter DJ, Torkelson JL, Bodnar J, Mortazavi B, Laurent T, Deason J, Thephavongsa K, Zhong J. The Rickettsia endosymbiont of Ixodes pacificus contains all the genes of de novo folate biosynthesis. PLoS One. 2015;10(12):e0144552. doi:10.1371/journal.pone.0144552. PMID: 26650541; PMCID: PMC4674097.

25. Duron O, Binetruy F, Noël V, et al. Evolutionary changes in symbiont community structure in ticks. Mol Ecol. 2017;26(11):2905-21. doi:10.1111/mec.14094.

26. Gerhart JG, Moses AS, Raghavan R. A Francisella-like endosymbiont in the Gulf Coast tick evolved from a mammalian pathogen. Sci Rep. 2016;6:33670. doi:10.1038/srep33670. PMID: 27645766; PMCID: PMC5028885.

27. Patrick CD, Hair JA. Laboratory rearing procedures and equipment for multi-host ticks (Acarina: Ixodidae). J Med Entomol. 1975;12(3):389 - 90. doi: 10.1093/jmedent/12.3.389. PMID: 1181449.

28. Karim S, Ribeiro JM. An insight into the sialome of the lone star tick, Amblyomma americanum, with a glimpse on its time dependent gene expression. PLoS One. 2015;10(7):e0131292. doi:10.1371/journal.pone.0131292. PMID: 26131772; PMCID: PMC4489193.

29. Klindworth A, Pruesse E, Schweer T, Peplies J, Quast C, Horn M, et al. Evaluation of general $16 \mathrm{~S}$ ribosomal RNA gene PCR primers for classical and next-generation sequencing-based diversity studies. Nucleic Acids Res. 2013;41(1):e1. doi:10.1093/nar/gks808.

30. Schirmer M, ljaz UZ, D'Amore R, Hall N, Sloan WT, Quince C. Insight into biases and sequencing errors for amplicon sequencing with the Illumina MiSeq platform. Nucleic Acids Res. 2015;43(6):e37. doi:10.1093/nar/gku1341. Epub 2015 Jan 13. PMID: 25586220; PMCID: PMC4381044.

31. Schloss PD, Gevers D, Westcott SL. Reducing the effects of PCR amplification and sequencing artifacts on 16S rRNA-based studies. PLoS One. 2011;6(12):e27310. 
doi:10.1371/journal.pone.0027310. Epub 2011 Dec 14. PMID: 22194782; PMCID: PMC3237409.

32. Rideout JR, Chase JH, Bolyen E, Ackermann G, González A, Knight R, Caporaso JG. GigaScience. Keemei: cloud-based validation of tabular bioinformatics file formats in Google Sheets 2016;5:27. http://dx.doi.org/10.1186/s13742-016-0133-6.

33. Bolyen E, Rideout JR, Dillon MR, Bokulich NA, Abnet CC, Al-Ghalith, et al. Reproducible, interactive, scalable and extensible microbiome data science using QIIME 2. Nat Biotechnol. 2019;37(8):852857. doi: 10.1038/s41587-019-0209-9. Erratum in: Nat Biotechnol. 2019;37(9):1091. PMID: 31341288; PMCID: PMC7015180.

34. Bokulich NA, Subramanian S, Faith JJ, Gevers D, Gordon JI, Knight R, et al. Quality-filtering vastly improves diversity estimates from Illumina amplicon sequencing. Nat Methods. 2013;10(1):57-9. doi:10.1038/nmeth.2276.

35. Katoh K, Standley DM. MAFFT multiple sequence alignment software version 7: improvements in performance and usability. Mol Biol Evol. 2013;30(4):772-80. doi:10.1093/molbev/mst010. Epub 2013 Jan 16. PMID: 23329690; PMCID: PMC3603318.

36. Price MN, Dehal PS, Arkin AP. FastTree 2-approximately maximum-likelihood trees for large alignments. PLoS ONE. 2010;5:e9490.

37. Quast C, Pruesse E, Yilmaz P, Gerken J, Schweer T, Yarza P, Peplies J, Glöckner FO. The SILVA ribosomal RNA gene database project: improved data processing and web-based tools. Nucleic Acids Res. 2013;41(Database issue):D590-6. doi: 10.1093/nar/gks1219. Epub 2012 Nov 28. PMID: $23193283 ;$ PMCID: PMC3531112.

38. Chong J, Liu P, Zhou G, Xia J. Using MicrobiomeAnalyst for comprehensive statistical, functional, and meta-analysis of microbiome data. Nat Protoc. 2020;15(3):799-821. doi:10.1038/s41596-0190264-1. Epub 2020 Jan 15. PMID: 31942082.

39. Dhariwal A, Chong J, Habib S, King IL, Agellon LB, Xia J. MicrobiomeAnalyst: a web-based tool for comprehensive statistical, visual and meta-analysis of microbiome data. Nucleic Acids Res. 2017;45(W1):W180-8. doi:10.1093/nar/gkx295. PMID: 28449106; PMCID: PMC5570177.

40. Friedman J, Alm EJ. Inferring correlation networks from genomic survey data. PLoS Comput Biol. 2012;8(9):e1002687. doi:10.1371/journal.pcbi.1002687. Epub 2012 Sep 20. PMID: 23028285; PMCID: PMC3447976.

41. Lozupone C, Knight R. UniFrac: a new phylogenetic method for comparing microbial communities. Appl Environ Microbiol. 2005;71(12):8228-35. doi:10.1128/AEM.71.12.8228-8235.2005. PMID: 16332807; PMCID: PMC1317376.

42. Vázquez-Baeza Y, Pirrung M, Gonzalez A, Knight R. EMPeror: a tool for visualizing high-throughput microbial community data. Gigascience. 2013;2(1):16. doi:10.1186/2047-217X-2-16. PMID: 24280061 ; PMCID: PMC4076506.

43. Mira A, Moran NA. Estimating population size and transmission bottlenecks in maternally transmitted endosymbiotic bacteria. Microb Ecol. 2002;44(2):137-43. doi:10.1007/s00248-0020012-9. Epub 2002 Jun 28. PMID: 12087426. 
44. Burgdorfer W, Hayes SF, Mavros AJ. Nonpathogenic Rickettsiae in Dermacentor andersoni: a limiting factor for the distribution of Rickettsia rickettsii. In: Burgdorferi W, Anacker RL, editors. Rickettsiae and Rickettsial Diseases. New York: Academic; 1981. pp. 585-94.

45. Niebylski ML, Peacock MG, Fischer ER, Porcella SF, Schwan TG. Characterization of an endosymbiont infecting wood ticks, Dermacentor andersoni, as a member of the genus Francisella. Appl Environ Microbiol. 1997;63(10):3933-40. doi:10.1128/aem.63.10.3933-3940.1997. PMID: 9327558; PMCID: PMC168705.

46. Institute of Medicine (US) Committee on Lyme Disease and Other Tick-Borne Diseases. The State of the Science. Critical needs and gaps in understanding prevention, amelioration, and resolution of Lyme and other tick-borne diseases: the short-term and long-term outcomes: workshop report. Washington (DC): National Academies Press (US); PMID: 21977545.

47. Ponnusamy L, Gonzalez A, Van Treuren W, Weiss S, Parobek CM, Juliano JJ, et al. Diversity of rickettsiales in the microbiome of the lone star tick, Amblyomma americanum. Appl Environ Microbiol. 2014;80: 354-9. doi: 10.1128/AEM.02987-13 PMID: 24162580.

48. Sun LV, Scoles GA, Fish D, O'Neill SL. Francisella-like endosymbionts of ticks. J Invertebr Pathol. 2000;76(4):301-3. doi: 10.1006/jipa.2000.4983. PMID: 11112376.

49. Scoles GA. Phylogenetic analysis of the Francisella-like endosymbionts of Dermacentorticks. J Med Entomol. 2004;41(3):277 - 86. doi: 10.1603/0022-2585-41.3.277. PMID: 15185926.

50. Zhong J, Jasinskas A, Barbour AG. Antibiotic treatment of the tick vector Amblyomma americanum reduced reproductive fitness. PLoS One. 2007;2:e405.

51. Vautrin E, Vavre F. Interactions between vertically transmitted symbionts: cooperation or conflict? Trends Microbiol. 2009;17(3):95-9. doi:10.1016/j.tim.2008.12.002. Epub 2009 Feb 21. PMID: 19230673.

52. Moran NA, McCutcheon JP, Nakabachi A. Genomics and evolution of heritable bacterial symbionts. Annu Rev Genet. 2008;42:165 - 90. doi: 10.1146/annurev.genet.41.110306.130119. PMID: 18983256.

53. Wernegreen JJ. Endosymbiosis. Curr Biol. 2012;22(14):R555-61. doi: 10.1016/j.cub.2012.06.010. PMID: 22835786.

54. Murrell A, Campbell NJ, Barker SC. A total-evidence phylogeny of ticks provides insights into the evolution of life cycles and biogeography. Mol Phylogenet Evol. 2001;21(2):244 - 58. doi: 10.1006/mpev.2001.1018. PMID: 11697919.

55. Goethert HK, Telford SR 3rd. A new Francisella (Beggiatiales: Francisellaceae) inquiline within Dermacentor variabilis Say (Acari: Ixodidae). J Med Entomol. 2005;42(3):502-5. doi: 10.1093/jmedent/42.3.502. PMID: 15962806.

56. Clayton KA, Gall CA, Mason KL, Scoles GA, Brayton KA. The characterization and manipulation of the bacterial microbiome of the Rocky Mountain wood tick, Dermacentor andersoni. Parasit Vectors. 2015;8:632. 
57. McCutcheon JP, Moran NA. Extreme genome reduction in symbiotic bacteria. Nat Rev Microbiol. 2011;10(1):13-26. doi: 10.1038/nrmicro2670. PMID: 22064560.

58. McNulty SN, Foster JM, Mitreva M, Dunning Hotopp JC, Martin J, Fischer K, et al. Endosymbiont DNA in endobacteria-free filarial nematodes indicates ancient horizontal genetic transfer. PLoS One. 2010;5(6):e11029. doi:10.1371/journal.pone.0011029. PMID: 20543958; PMCID: PMC2882956.

59. Gulia-Nuss M, Nuss AB, Meyer JM, Sonenshine DE, Roe RM, Waterhouse RM, et al. Genomic insights into the Ixodes scapularis tick vector of Lyme disease. Nat Commun. 2016;7:10507. doi:10.1038/ncomms10507. PMID: 26856261; PMCID: PMC4748124.

60. Bonnet SI, Binetruy F, Hernández-Jarguín AM, Duron O. The tick microbiome: why non-pathogenic microorganisms matter in tick biology and pathogen transmission. Front Cell Infect Microbiol. 2017;7:236. doi:10.3389/fcimb.2017.00236. PMID: 28642842; PMCID: PMC5462901.

61. Ivanov IN, Mitkova N, Reye AL, Hübschen JM, Vatcheva-Dobrevska RS, Dobreva EG, Kantardjiev TV, Muller CP. Detection of new Francisella-like tick endosymbionts in Hyalomma spp. and Rhipicephalus spp. (Acari: Ixodidae) from Bulgaria. Appl Environ Microbiol. 2011;77(15):5562-5. doi: 10.1128/AEM.02934-10. Epub 2011 Jun 24. PMID: 21705542; PMCID: PMC3147446.

62. Wójcik-Fatla A, Zając V, Sawczyn A, Cisak E, Dutkiewicz J. Babesia spp. in questing ticks from eastern Poland: prevalence and species diversity. Parasitol Res. 2015;114(8):3111-6. doi: 10.1007/s00436-015-4529-5. Epub 2015 May 16. PMID: 25976982; PMCID: PMC4513193.

63. Rounds MA, Crowder CD, Matthews HE, Philipson CA, Scoles GA, Ecker DJ, Schutzer SE, Eshoo MW. Identification of endosymbionts in ticks by broad-range polymerase chain reaction and electrospray ionization mass spectrometry. J Med Entomol. 2012;49(4):843-50. doi:10.1603/me12038. PMID: $22897044 ;$ PMCID: PMC3535486.

64. Gurfield N, Grewal S, Cua LS, Torres PJ, Kelley ST. Endosymbiont interference and microbial diversity of the Pacific coast tick, Dermacentor occidentalis, in San Diego County, California. PeerJ. 2017;5:e3202. doi: 10.7717/peerj.3202. PMID: 28503372; PMCID: PMC5426561.

65. Gall CA, Reif KE, Scoles GA, Mason KL, Mousel M, Noh SM, Brayton KA. The bacterial microbiome of Dermacentor andersoni ticks influences pathogen susceptibility. ISME J. 2016;10(8):1846-55. doi:10.1038/ismej.2015.266. Epub 2016 Feb 16. PMID: 26882265; PMCID: PMC5029153.

66. Lejal E, Chiquet J, Aubert J, Robin S, Estrada-Peña A, Rue O, et al. Temporal patterns in Ixodes ricinus microbial communities: an insight into tick-borne microbe interactions. Microbiome. 2021;9(1):153. doi:10.1186/s40168-021-01051-8. PMID: 34217365; PMCID: PMC8254910.

67. Carpi G, Cagnacci F, Wittekindt NE, Zhao F, Qi J, Tomsho LP, et al. Metagenomic profile of the bacterial communities associated with Ixodes ricinus ticks. PLoS One. 2011;6(10):e25604. doi:10.1371/journal.pone.0025604. Epub 2011 Oct 13. PMID: 22022422; PMCID: PMC3192763.

68. 3. Sharma SR, Karim S. Tick saliva and the Alpha-Gal Syndrome: finding a needle in a haystack. Front Cell Infect Microbiol 11:680264. doi: 10.3389/fcimb.2021.680264.

69. Cabezas-Cruz A, Estrada-Peña A, de la Fuente J. The good, the bad and the tick. Front Cell Dev Biol. 2019;7:79. doi:10.3389/fcell.2019.00079. PMID: 31157221; PMCID: PMC6529820. 
70. Montassier E, Al-Ghalith GA, Mathé C, Le Bastard Q, Douillard V, Garnier A, et al. Distribution of bacterial a1,3-galactosyltransferase genes in the human gut microbiome. Front Immunol. 2020;10:3000. doi:10.3389/fimmu.2019.03000. PMID: 31998300; PMCID: PMC6970434.

71. Brown DB, Muszynski A, Carlson RW. Elucidation of a novel lipid A a-(1,1)-GalA transferase gene ( $\mathrm{rgtF}$ ) from Mesorhizobium loti: heterologous expression of $\mathrm{rgtF}$ causes Rhizobium etli to synthesize lipid A with a-(1,1)-GalA. Glycobiology. 2013;23(5):546-558. DOI: 10.1093/glycob/cws223.

72. Ben-Yosef M, Rot A, Mahagna M, Kapri E, Behar A, Gottlieb Y. Coxiella-like endosymbiont of Rhipicephalus sanguineus is required for physiological processes during ontogeny. Front Microbiol. 2020;11:493. doi:10.3389/fmicb.2020.00493. PMID: 32390951; PMCID: PMC7188774.

\section{Figures}

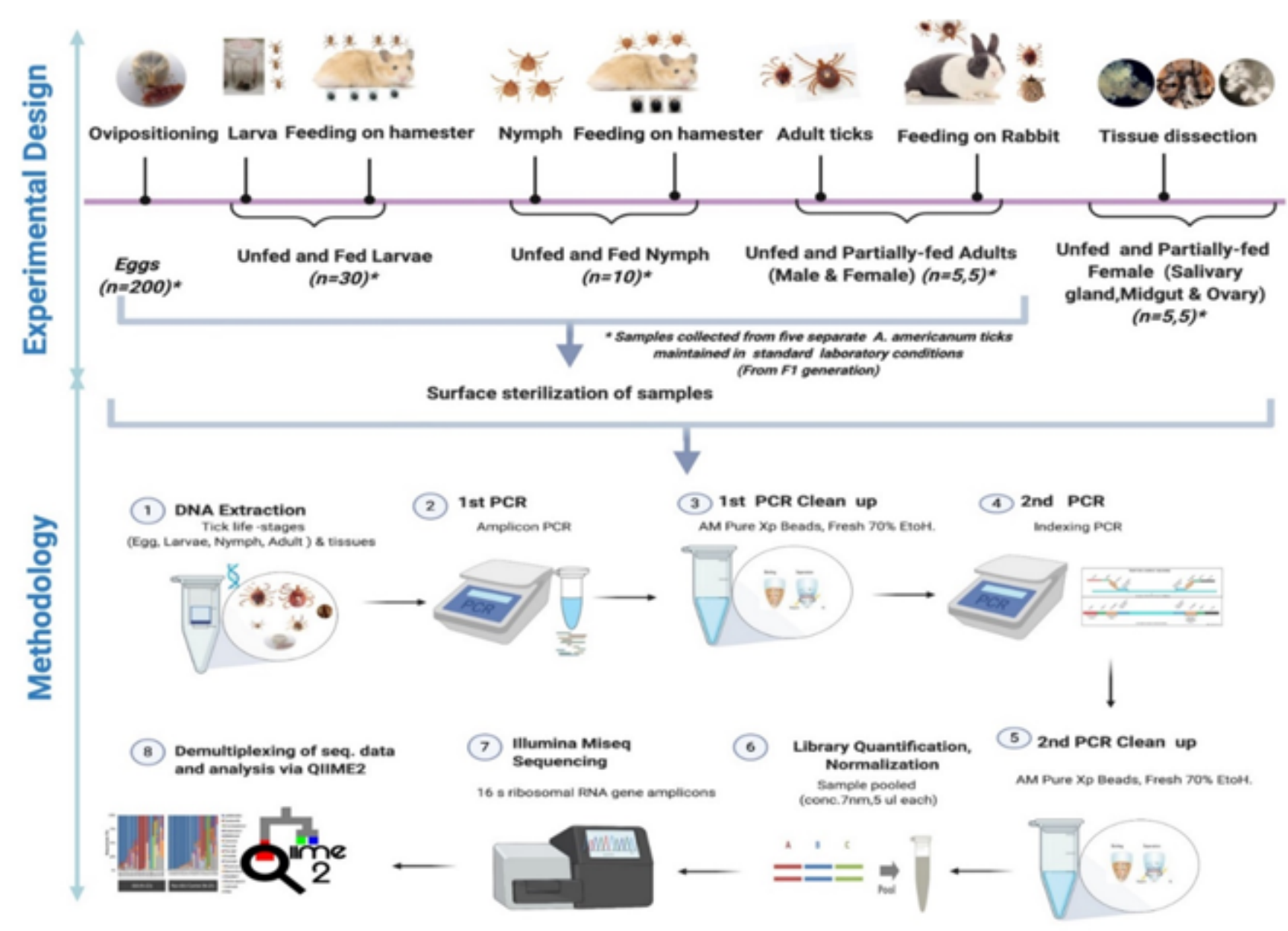

Figure 1

Schematic workflow of microbiome study of the lone star tick for its life stages and tissues. 

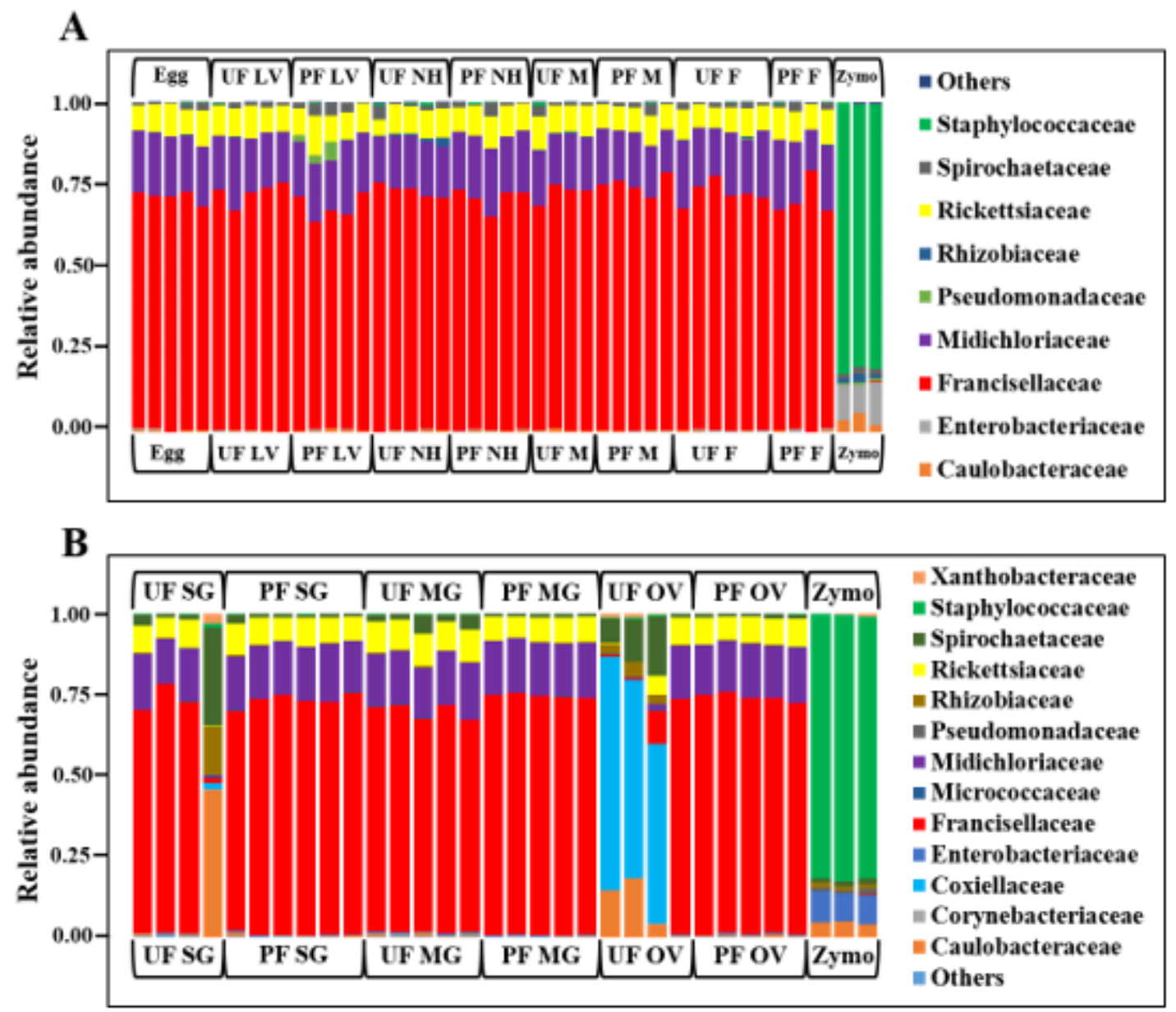

Figure 2

Relative abundances of bacteria profiles showing the topmost abundant bacteria families to the right of the graphs. Each bar represents a single biological replicate from different A) developmental stages and B) isolated tissue from lab raise unfed (UF) and partially-fed (PF) Amblyomma americanum ticks. The family Francisellaceae is the most dominant group occurring at an abundance of $62 \%-80 \%$ across the tick developmental stages (Fig. A) and 10\%-77\% across isolated tissues irrespective of the blood meal. The family Coxiellaceae was detected in 3 of the 4 replicates from the unfed ovarian tissues (UF OV) at a relative abundance of 55\%-72\% (Fig. B). (UF; unfed, PF; partially-fed, LV; larvae, NH; nymph, M; male, FM; female, SG; salivary gland, MG; midgut, OV; ovary, Zymo; positive bacteria control). 

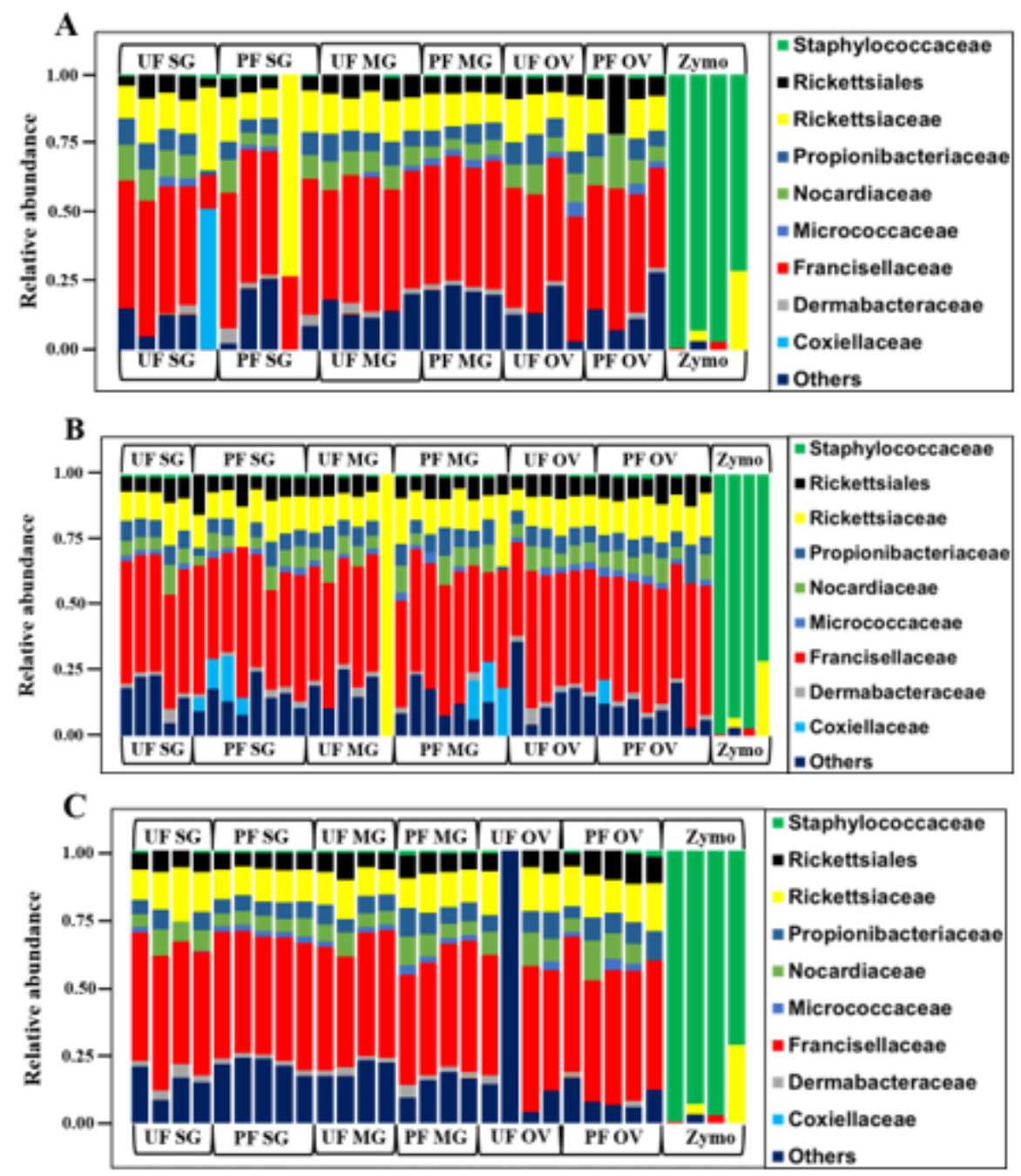

Figure 3

Relative abundances of bacteria profiles showing the topmost abundant bacteria families to the right of the graphs. Each column represents a single replicate from different tissues isolated from unfed (UF) and partially-fed (PF) Amblyomma americanum ticks collected from A) Illinois, B) Delaware, and C) Maryland. All ticks displayed a similar microbial profile with Francisellaceae being the most abundant family (26\%-60\%). The family Nocardiaceae, Rickettsiaceae, Propionibacteriaceae, and Rickettsiales were also present in relatively equal abundance in ticks from all three geographical locations. Three individual replicate each belonging to the partially-fed salivary gland and midgut (PF SG and PF MG) from Delaware both have Coxiellaceae identified in their microbial composition. (UF; unfed, PF; partially-fed, SG; salivary gland, MG; midgut, OV; ovary, Zymo; positive bacteria control) 


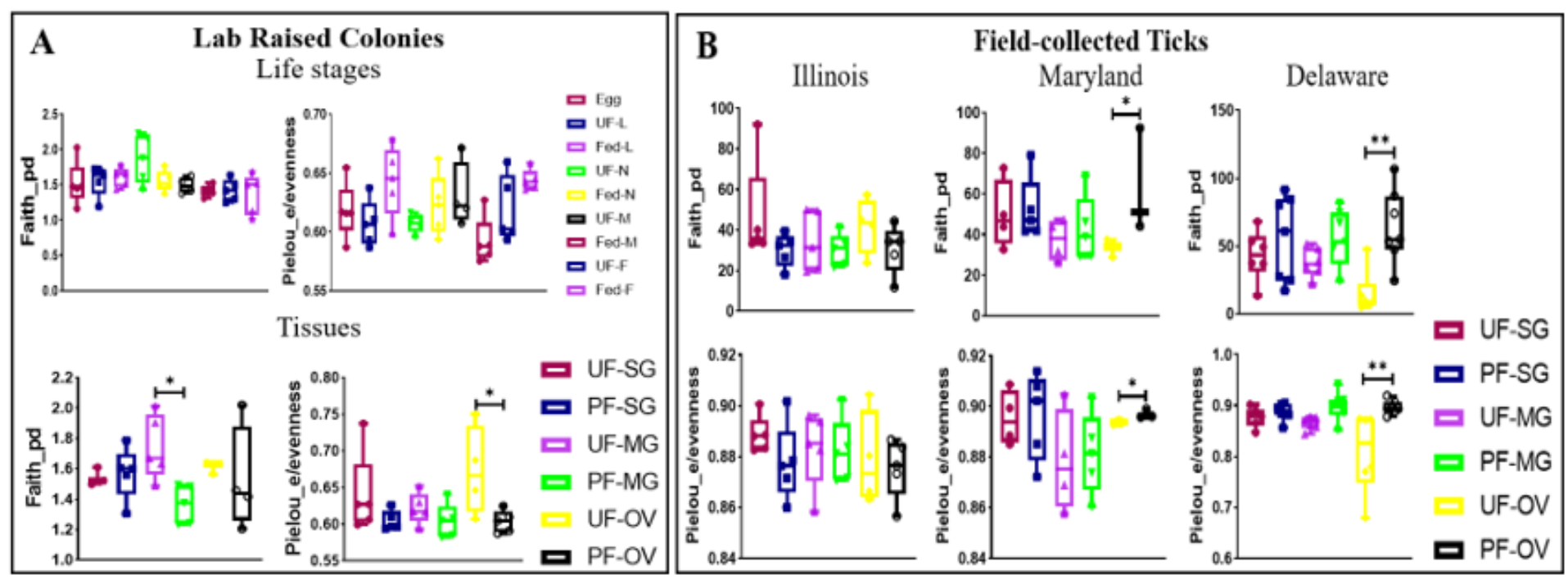

Figure 4

Alpha diversity metrics. Richness (Faith_pd) and evenness (Pielou_e) index for A) lab raised tick tissues and developmental stages, and B) field collected tick tissues. Each point on the box plot represents an individual tick or tissue replicate. Asterisks designate significance level as follows $* \star \star \leq 0.001, * \star \leq 0.01$, ${ }^{*} \leq 0.05$.
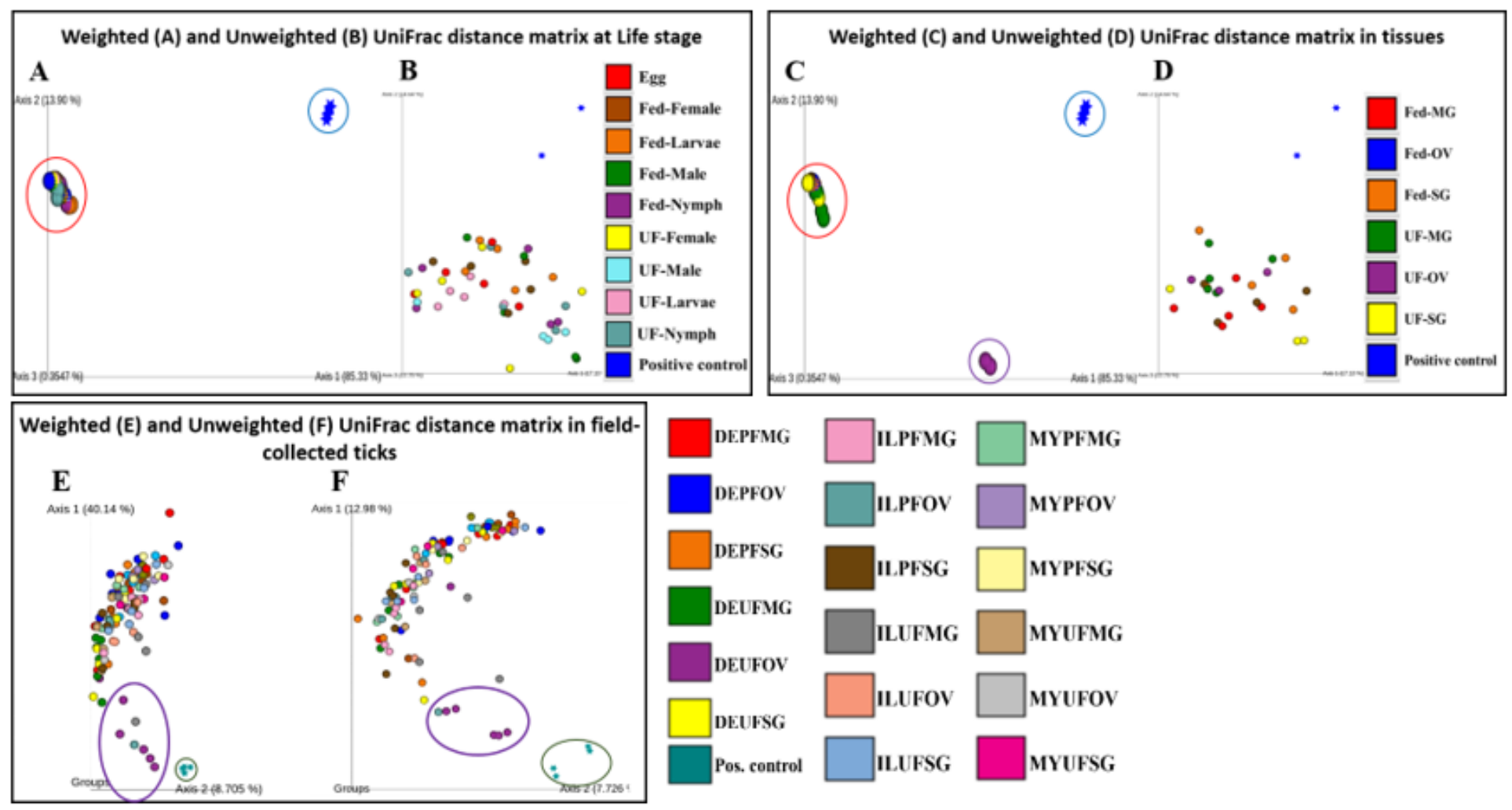

\section{Figure 5}

Beta diversity metrics. Emperor plot of PCoA analysis of weighted and unweighted UniFrac distance dissimilarity measures of microbial composition. Each colored ellipse corresponds to uniquely clustering 
patterns of biological replicates.
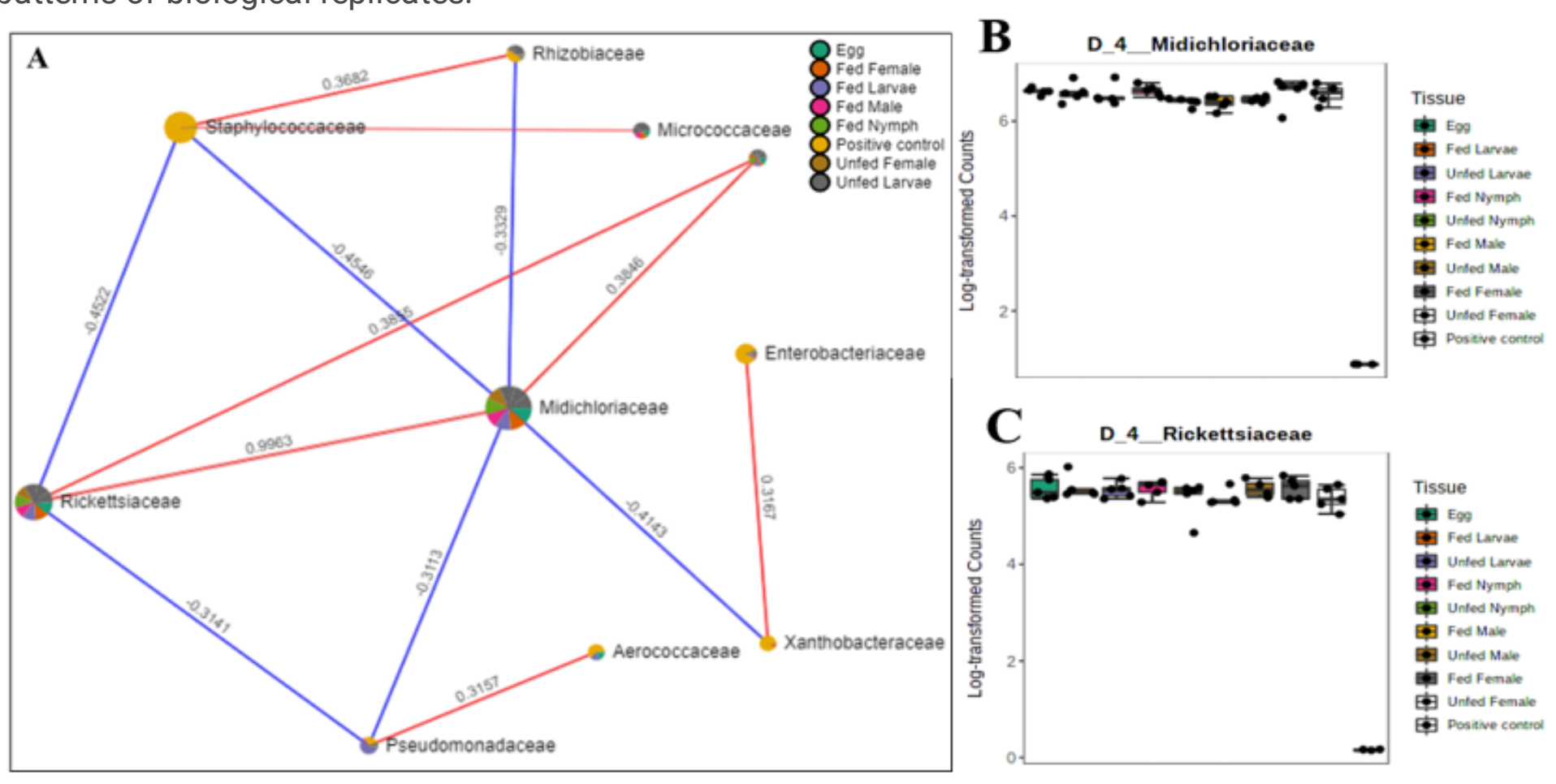

\section{Figure 6}

Correlation network analysis in different life stages of lab-raised Amblyomma americanum ticks.

Correlation network generated using the SparCC algorithm. Correlation network with nodes representing taxa at the family level and edges representing correlations between taxa pairs A) and representative boxplots showing enriched abundance of Midichloriaceae B), and Rickettsiaceae C) across all samples. 

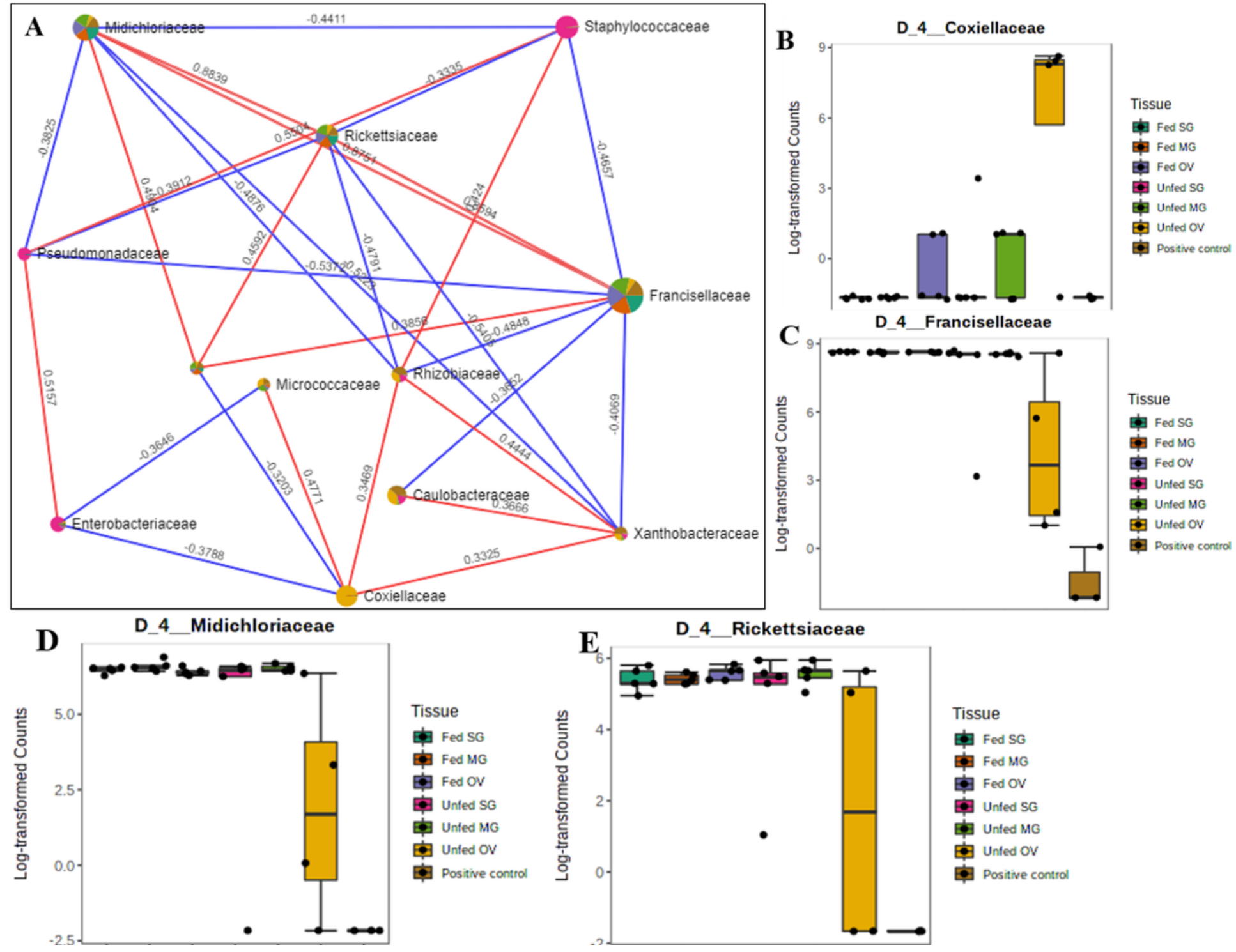

Tissue

审 Fed SG

审 Fed MG

审 Fed OV

审 Unfed SG

闹 Unfed MG

审 Unfed ov

审 Positive control

Figure 7

Correlation network analysis in different tissues of lab-raised Amblyomma americanum ticks. Correlation network generated using the SparCC algorithm. Correlation network with nodes representing taxa at the family level and edges representing correlations between taxa pairs A) and representative boxplots showing enriched abundance of Coxiellaceae B), Midichloriaceae B), and Rickettsiaceae C) across all samples. 

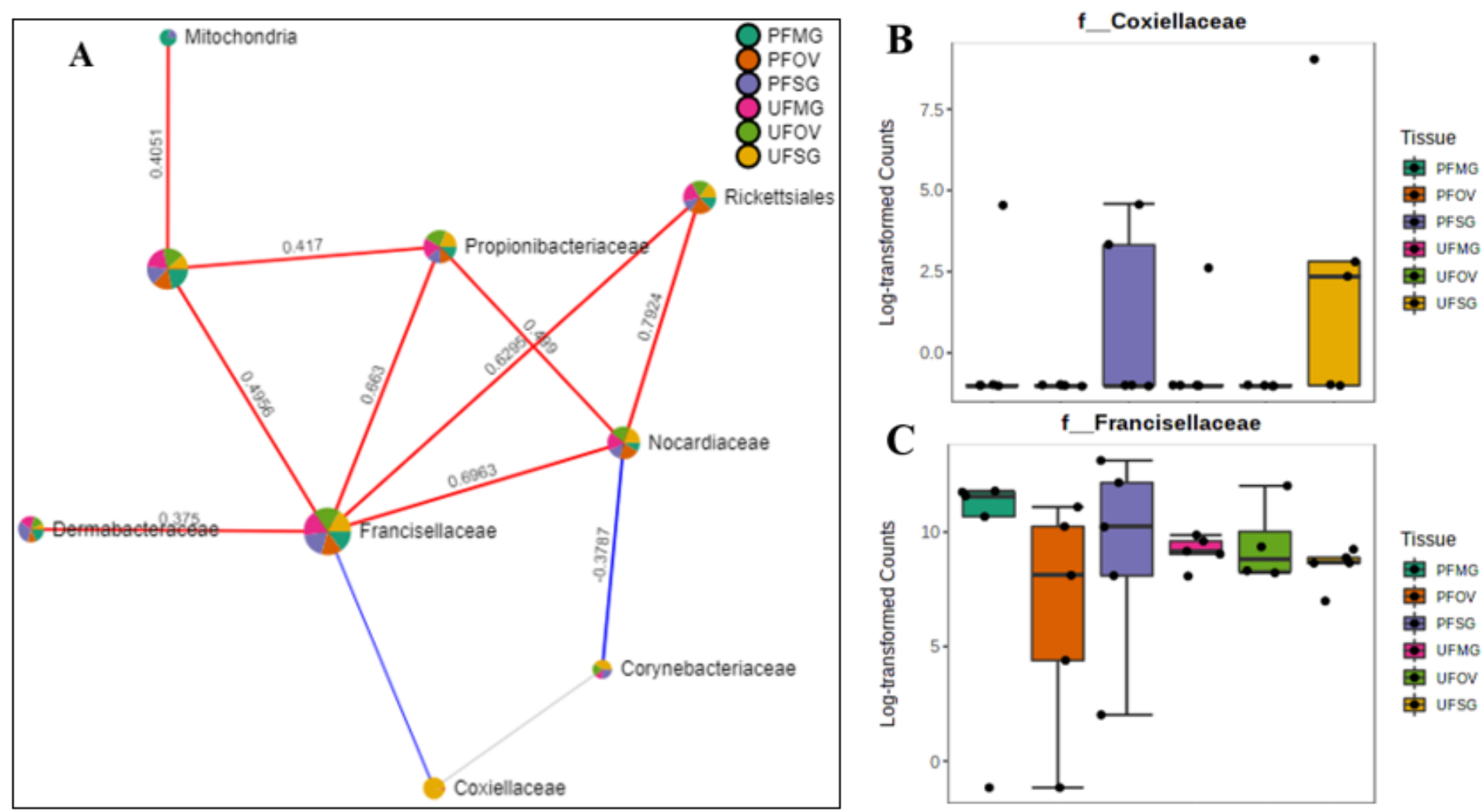

Figure 8

Correlation network analysis in different tissues of Amblyomma americanum ticks from Illinois. Correlation network generated using the SparCC algorithm. Correlation network with nodes representing taxa at the family level and edges representing correlations between taxa pairs A) and representative boxplots showing enriched abundance of Coxiellaceae B), Francisellaceae B) across all samples. 

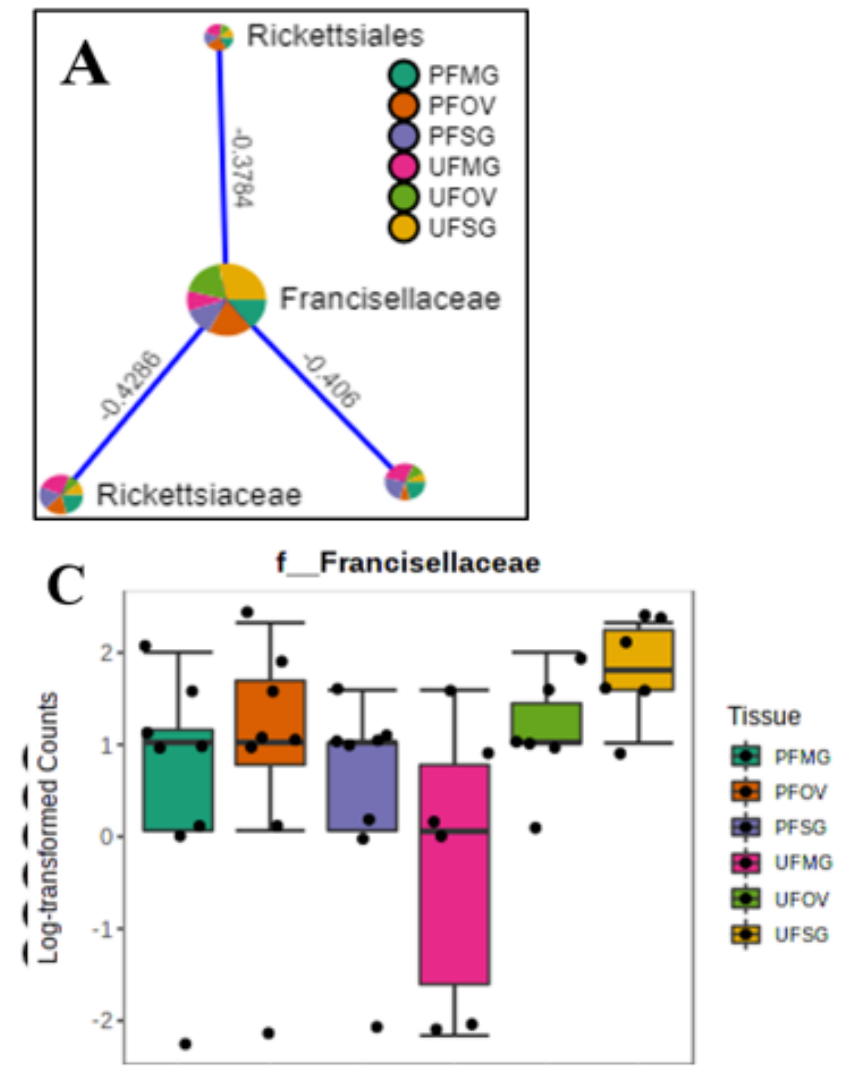
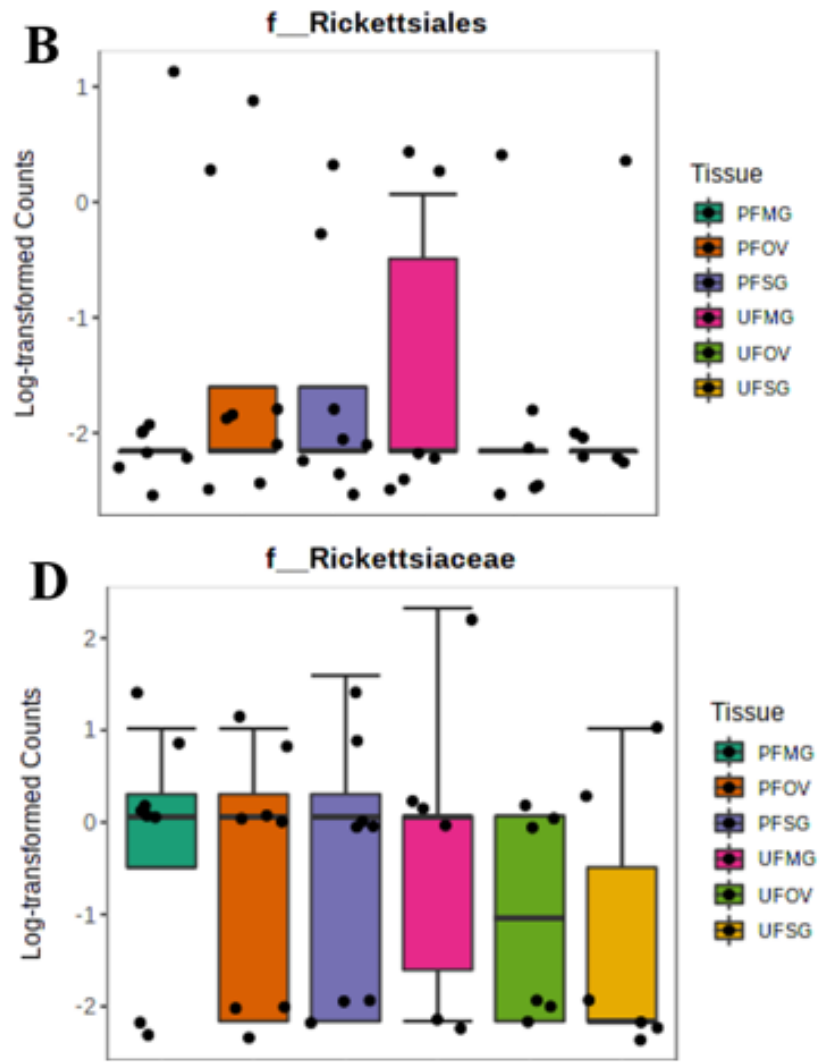

Figure 9

Correlation network analysis in different tissues of ticks from Delaware. Correlation network generated using the SparCC algorithm. Correlation network with nodes representing taxa at the family level and edges representing correlations between taxa pairs $A$ ) and representative boxplots showing enriched abundance of Rickettsiales B), Francisellaceae B), and Rickettsiaceae C) across all samples. 


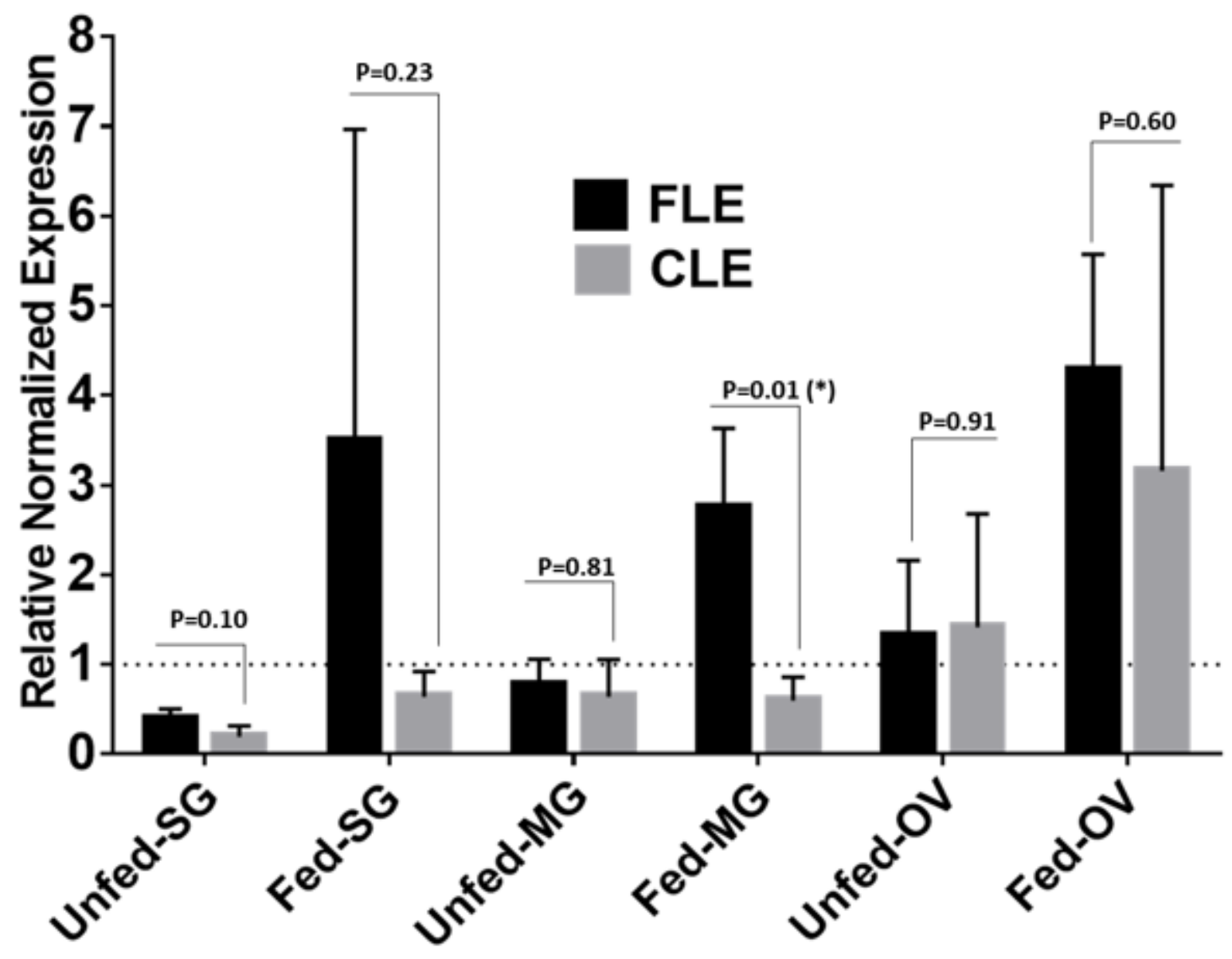

Figure 10

qPCR-based relative quantification of FLE and CLE in field collected tick tissues (salivary gland, midgut and ovary) from Delaware. All the expressions shown are relative to actin. Expression of actin has been shown as 1 (dotted line). Statistically significant comparisons are indicated by * $(p \leq 0.05)$. SG-salivary gland, MG-midgut, OV-ovary.

\section{Supplementary Files}

This is a list of supplementary files associated with this preprint. Click to download.

- Supplement.docx 